\title{
Encoding of odor fear memories in the mouse olfactory cortex
}

Claire Meissner-Bernard ${ }^{1,2}$, Yulia Dembitskaya ${ }^{1}$, Laurent Venance ${ }^{1}$, and Alexander Fleischmann ${ }^{1,3^{*}}$

${ }^{1}$ Center for Interdisciplinary Research in Biology (CIRB), Collège de France, and CNRS UMR 7241 and INSERM U1050, Paris, France

${ }^{2}$ current address: Friedrich Miescher Institute for Biomedical Research, 4058 Basel, Switzerland

${ }^{3}$ Department of Neuroscience, and the Robert J. and Nancy D. Carney Institute for Brain Science, Brown University, Providence, RI 02912, USA

*Corresponding author and lead contact: alexander_fleischmann@brown.edu 


\section{Summary}

Odor memories are exceptionally robust and essential for animal survival. The olfactory (piriform) cortex has long been hypothesized to encode odor memories, yet the cellular substrates for olfactory learning and memory remain unknown. Here, using intersectional, cFos-based genetic manipulations ("Fos-tagging"), we show that olfactory fear conditioning activates sparse and distributed ensembles of neurons in mouse piriform cortex. We demonstrate that chemogenetic silencing of these Fostagged piriform ensembles selectively interferes with odor fear memory retrieval, but does not compromise basic odor detection and discrimination. Furthermore, chemogenetic reactivation of piriform neurons that were Fos-tagged during olfactory fear conditioning causes a decrease in exploratory behavior, mimicking odor-evoked fear memory recall. Together, our experiments identify specific ensembles of piriform neurons as critical components of an olfactory fear memory trace. 


\section{Introduction}

Odor perception and behavioral responses to odors strongly depend on experience, and learned odor-context associations often last for the lifetime of an animal [1]. The cellular and neural circuit mechanisms underlying olfactory learning and memory, however, remain poorly understood. Recent studies on episodic and contextual learning in hippocampus and cortex have suggested that memories are encoded in distributed ensembles of neurons, often referred to as a 'memory trace' or 'engram' $[2,3]$. These engram cells are thought to store information about the environmental context and associated emotions of past experiences, and their activity is necessary and sufficient for memory recall [4-6].

Here, we investigate the organization of odor memories in the olfactory (piriform) cortex of mice. The piriform cortex (PCx), a trilaminar paleocortical structure, is the largest cortical area receiving direct afferent inputs from the olfactory bulb, which, in turn, receives topographically organized inputs from olfactory sensory neurons in the nose. Individual piriform neurons respond to combinatorial inputs from multiple olfactory bulb projection neurons $[7,8]$, suggesting that odor objects are constructed in PCx from the molecular features of odorants extracted in the periphery [9]. PCx has long been hypothesized to support auto-associative network functions that can retrieve previously learned information from partial or degraded sensory inputs $[10,11]$. Piriform pyramidal cells form a large recurrent network, which is reciprocally connected with high-order associative areas including the prefrontal, entorhinal and perirhinal cortex, and the amygdala $[12,13]$. Storage of information is made possible

by NMDA-dependent, associative plasticity of connections [14-16]. Furthermore, changes in piriform network activity [17-19] and stabilization of odor representations 
[20] have been observed after associative olfactory learning. Finally, excitotoxic lesions of the posterior PCx in rats perturb odor fear memories [21], and optogenetic stimulation of artificial piriform ensembles is sufficient to drive learned behaviors [22]. Taken together, these studies have led to the hypothesis that piriform neural ensembles encode olfactory memory traces.

We have adapted an intersectional genetic strategy to target components of odor fear memories in the PCx. We combined cFos-tTA transgenic mice [23], in which the ITA transcription factor is expressed under the promoter of the immediate early gene cFos, with virally targeted expression of tTA-responsive fluorescent reporters and regulators of neural activity [24-26]. This approach allows us to selectively and persistently "Fos-tag" piriform neurons that were activated during olfactory fear conditioning. We then tested the behavioral consequences of manipulating such Fostagged piriform ensembles during memory recall. We find that chemogenetic silencing of Fos-tagged piriform ensembles interferes with a learned odor escape behavior. In contrast, silencing piriform neurons Fos-tagged during presentation of a neutral odor only partially attenuates odor fear memory recall, suggesting that memory impairments are largely specific to the piriform ensemble that is Fos-tagged during learning. Finally, artificial reactivation of Fos-tagged piriform neurons elicits behavioral changes consistent with memory recall. Together, our results suggest that piriform neural ensembles encode an essential component of olfactory fear memories. 


\section{Results}

\section{Behavioral expression of odor fear memory depends on the retrieval context}

We used classical conditioning to train mice to associate an odor with foot shock (the reinforced conditioned stimulus, CS+) [22]. Training occurred in a rectangular box with odor ports at each extremity. Brief odor stimuli $(7 \mathrm{~s})$ were presented on randomly alternating sides when mice were close to the odor port. The CS+ (ethyl acetate) was paired with a foot shock, applied to the side of the box where the odor was delivered. Mice escaped from the foot shock by running towards the opposite side of the box (see Methods and Figure 1A). Two control odors, whose presentation was not paired with foot shock (non-reinforced stimuli, CS-), were used to entrain specific as opposed to generalized fear responses to odors [18]. Memory retrieval of cFos-tTA transgenic mice $(n=9)$ was first tested by presenting the odors alone in a similar context as during conditioning: same stimulus duration and a testing box with the same dimensions as the training box but with a different texture and color (see Methods). We combined two parameters to quantify the learned escape behavior: the position of the mouse in the box and the increase in its maximum velocity after odor presentation (Figure 1B). These parameters were chosen because they described well the behavioral response of mice to the odor-foot shock pairing during training (Figure S1). The median position of the mice in the box at the end of stimulus delivery was 0.71 (with 0 defined as the site of odor presentation and 1 as the opposite end of the box), and mice exhibited a 1.8-fold increase of their maximum velocity. Importantly, the escape behavior was specific to the CS+, as none of the mice ran away from the CS- (Figure 1C, D and E). We then subtracted the position after CS- presentation from the one after CS+ presentation and the CS- maximum velocity ratio (after/before presentation) from the CS+ one. A value of 0 would 
indicate a generalized learned response to odors (escape from the CS+ and the CS-) or no learning. Here, the differences were significantly different from 0 (Wilcoxon Signed-Rank Test (WSRT), $\mathrm{W}=45, \mathrm{p}=0.0039$ for both the position and velocity ratio, Figure $1 F$ and $G)$.

During both training and testing, odors were presented at the extremities of the box, and the training and testing box have the same shape and dimensions. Therefore, contextual features (visual and/or tactile) could be an important determinant for successful memory retrieval. To assess the context-dependence of the memory, we tested memory retrieval in a different context. We monitored mouse behavior in an open field during ambient and prolonged odor exposure, where the odor does not originate from a spatially defined odor source as during training (Figure 1A). We fear conditioned mice to ethyl acetate as described above $(n=6)$. Mice exposed to ethyl acetate without foot shock served as a control $(n=5)$. Diffusion of ethyl acetate above the open field caused a significant decrease in exploratory behavior of fearconditioned mice compared to control mice (Mann-Whitney test (MWT), $U=0$, $\mathrm{p}=0.0043$, Figure $1 \mathrm{H}$ and I). Baseline exploratory behavior measured one day earlier did not differ between fear-conditioned and control mice (MWT, $U=11, p=0.5368$ ). These data suggest that re-exposure of mice to the CS+ induced memory recall, expressed as a decrease in exploratory behavior in the open field test. Similar results were obtained when mice where trained only with a CS+ and no CS- (MWT, $U=2$, $p=0.0173$ for ratio, Figure $\mathbf{1} \mathbf{J}$ and $\mathbf{K})$. The differences in behavioral responses we observe in the two contexts (escape versus decreased exploratory behavior) likely reflects the adaptivity of fear [27].

Fos-expressing piriform neurons can be tagged and functionally manipulated 
If piriform neurons that were activated during olfactory learning encode an essential component of olfactory memories, then manipulating the activity of these neurons should impact memory recall.

As the PCx is a large area, we used Designer Receptors Exclusively Activated by Designer Drugs (DREADDs) $[24,25]$ to manipulate its activity. DREADDs increase (hM3Dq:mCherry) or decrease (HA:hM4Di-IRES-mCitrine) the excitability of neurons upon activation by their ligand clozapine $\mathrm{N}$-oxide (CNO). To selectively label piriform neurons that were activated during odor exposure, we used cFos-tTA transgenic mice [5] in which the activity-dependent cFos promoter drives expression of the tTA transcription factor, and we stereotaxically injected in PCx Adeno-Associated Viruses (AAVs) expressing DREADDs under the control of the tTA-responsive promoter tetO (Figure 2A). Temporal control of DREADD expression is provided by doxycycline, which interferes with the binding of tTA to tetO and thus suppresses transgene expression [28]. Mice maintained in their home cage on a doxycycline-containing diet showed low basal DREADD expression, detected by anti-HA immunohistochemistry and native mCherry fluorescence (Supplementary Figure 3C). To induce DREADD expression, mice were taken off doxycycline five days before odor presentation. Previous studies have shown that cFos-tTA-dependent-dependent expression of regulators of neural activity lasts for at least five days $[4,26,29]$. We therefore restricted our manipulations of neural activity to a three-day time window after Fostagging. Exposure to odor followed by foot shock resulted in the Fos-tagging of sparse neural ensembles (median (interquartile range): $1.6(0.3) \%$ of piriform neurons, $\mathrm{n}=2$ for $\mathrm{hM} 4 \mathrm{Di} ; 2.2(0.5) \%$ of piriform neurons, $\mathrm{n}=3$ for $\mathrm{hM} 3 \mathrm{Dq})$. Fos-tagged neurons were broadly dispersed throughout the anterior and posterior PCx (Figure 
2C and D and Figure S3). The number and distribution of Fos-tagged cells were similar to endogenous Fos expression $(2.9(0.2) \%, n=2$, Figure 2B and $[30,31])$. To test whether CNO-mediated activation of DREADDs in Fos-tagged piriform neurons alters their excitability, we performed whole-cell recordings in acute brain slices. DREADD-expressing neurons were identified based on mCitrine (HA:hM4DiIRES-mCitrine) or mCherry (hM3Dq:mCherry) fluorescence. Fos-tagged neurons displayed diverse morphologies and electrophysiological properties and included both excitatory (pyramidal-like neurons, PN; semilunar-like neurons, SL) and inhibitory neurons (fast-spiking interneurons, FS, Figure S2). In the absence of CNO, the resting membrane potential of DREADD-negative neurons was indistinguishable from DREADD-expressing neurons (-72.14 (2.96) $\mathrm{mV}$ in DREADD-negative neurons, $\mathrm{n}=17 ;-71.58$ (4.50) $\mathrm{mV}$ in hM4Di-expressing neurons, $\mathrm{n}=12 ;-72.56$ (4.18) $\mathrm{mV}$ in hM3Dq-expressing neurons, $n=12 ; p=0.819$, Kruskal-Wallis ANOVA, Figure 2E). After bath application of CNO $(5 \mu \mathrm{M})$, the resting membrane potential of DREADDnegative cells remained unchanged (WSRT, -72.27 (3.72) $\mathrm{mV}, \mathrm{n}=17, \mathrm{p}=0.818$ ), whereas it changed in DREADD-expressing cells: hM4Di-expressing cells hyperpolarized and hM3Dq-expressing cells depolarized (WSRTs, -74.51 (5.17) mV, $\mathrm{n}=12, \mathrm{p}=0.011$ and -66.95 (5.29) $\mathrm{mV}, \mathrm{n}=12, \mathrm{p}=0.005$, respectively; Figure $2 \mathrm{E})$. To determine the impact of $\mathrm{CNO}$ treatment on neuronal excitability, we delivered depolarizing current steps and compared the number of action potentials triggered by 60 pA step current above action potential threshold (see Methods and Figure 2G). We found that in hM4Di-expressing cells, the number of evoked action potentials significantly decreased after CNO application, whereas in hM3Dq-expressing cells, it significantly increased (WSRTs: 3.75 (1.59) vs. 0.30 (3.48), $n=10, p=0.010$ and 8.67 (9.10) vs. 19.0 (27.6) $\mathrm{mV}, \mathrm{n}=11, \mathrm{p}=0.006$, respectively). In contrast, DREADD- 
negative cells did not exhibit a significant change in the number of evoked action potentials upon CNO application (4.33 (3.88) vs. 4.67 (5.08), $n=11, p=0.865$, WSRT, Figure 2H).

Performing One Sample Wilcoxon Signed Rank Tests on the membrane potential and the number of action potentials after CNO application normalized to baseline values (in \%) gave similar p-values (Figure 2F and I). While CNO effects were similar in different cell types (FS, PN, SL), sample sizes are insufficient to quantify or exclude cell type-dependent variability.

Taken together, these experiments show that Fos-tagging during olfactory fear conditioning marks a sparse and dispersed subpopulation of piriform neurons, and that CNO-mediated activation of hM4Di or hM3Dq expressed in Fos-tagged neurons selectively decreases or increases their excitability.

\section{Silencing Fos-tagged piriform ensembles impairs odor fear memory recall}

To test the effects of Fos-tagged ensemble manipulation on memory recall, we Fostagged piriform neurons during olfactory fear conditioning, and then chemogenetically silenced them during memory recall. cFos-tTA male mice ("experimental group", n=8) were bilaterally injected in PCx with the AAV-tetO-hM4Di vector. Wild-type male mice injected with the same virus served as a control ("control group", $n=8$ ). Doxycycline was removed from the diet of mice prior to training, to permit the induction of hM4Di expression in Fos-expressing piriform neurons of mice of the experimental group. Mice were returned to doxycycline-containing diet immediately after odor fear conditioning, and memory recall of the odor-foot shock association was tested three days later in the rectangular testing box after intraperitoneal injection of CNO. (Figure 3A). Mice from the control group also received CNO injection, to control for 
behavioral changes caused by potential off-target $\mathrm{CNO}$ effects [32]. For all mice, we verified viral gene expression by post hoc histological examination. Previous studies have suggested that the posterior PCx is important for associative memory encoding $[10,21,33]$. We therefore excluded from our analysis mice in which parts of the posterior PCx were spared from viral infection (Figures S3 and S4A).

As expected, control mice exhibited robust escape behavior upon CS+ presentation (median position in the box at the end of CS+ delivery:0.74; increase in maximum velocity:1.9-fold, Figure 3B, C and E). In contrast, escape behavior was significantly reduced in mice in which Fos-tagged neurons were silenced. Their median position in the box at the end of stimulus delivery (0.42) and their increase in maximum velocity (1.1-fold) were significantly lower than in the control group (MWT, $U=7, p=0.007$ for both position and velocity, Figure 3B, C and E). Subtracting the CS- position and maximum velocity ratio from the $\mathrm{CS}_{+}$ones also lead to significant differences between the control and experimental groups (MWT, $U=13, p=0.0499$ for position and $U=11, p=0.0281$ for maximum velocity ratio, Figure 3D and $G$ ). Differences in behavioral responses of cFos-tTA mice were specifically due to hM4Di activation, as escape behavior after CNO injection was unaffected in cFos-tTA mice injected with AAVs expressing the calcium indicator GCaMP (Figure 1C, D, E and F).

Furthermore, all mice were similarly close to the odor port at the onset of CS+ odor presentation (MWT, $\mathrm{U}=28, \mathrm{p}=0.7209$, Figure 3B), and had similar maximum velocities during the $7 \mathrm{~s}$ time period that preceded CS+ presentation (MWT, $\mathrm{U}=24$, $p=0.4418$, Figure 3E). These observations exclude any inherent differences between groups due to how the CS+ was presented. Finally, we did not observe any differences in the overall mobility of mice throughout the entire testing session. The median speed of mice was not significantly different between the control and 
experimental group $(0.68(0.28) \mathrm{cm} / \mathrm{s}$ and $0.46(0.14) \mathrm{cm} / \mathrm{s}$ respectively, MWT, $\mathrm{U}=22$, $\mathrm{p}=0.3282$ ), nor did mice show a bias for the left or right side of the testing box (time spent on the left side divided by time spent on the right side: $1.36(0.72)$ and 1.51 (0.52) for the control and experimental groups respectively, MWT, $U=27, p=0.6454$ ). Together, these data suggest that Fos-tagged piriform ensembles that were activated during olfactory fear conditioning are necessary for robust odor fear memory recall. Even though cFos-tTA transgenic mice expressing hM4Di failed to robustly escape from the $\mathrm{CS}_{+}$, it should be noted that they behaved differently after CS+ and CSpresentation. They were further away from the odor port after CS+ presentation than after CS- presentation (WSRT, $\mathrm{W}=-36, \mathrm{p}=0.0078$, Figure $3 \mathrm{C}$ ), and their maximum velocity ratio was significantly higher (after/before odor presentation, WSRT, W=-36, $p=0.0078$, Figure $3 E, F$ and $\mathbf{G}$ ). This could indicate a partial memory of the learned association, possibly due to incomplete silencing of functionally relevant neurons in PCx, or the existence of parallel neural pathways that partially compensate for the loss of PCx functions.

\section{Memory impairments are specific to the piriform ensemble Fos-tagged during learning}

Silencing the PCx in only one brain hemisphere did not abolish the learned escape behavior to the conditioned odor (Figure S5). Furthermore, memory impairments were not related to the bilateral or unilateral infection of brain regions adjacent to PCx due to spillover of the virus (parts of cortical and basolateral amygdala, and insular cortex; Figure S5). Therefore, memory impairments are most likely specific to PCx. We next wanted to determine if the memory deficits were specific to the neurons that were Fos-tagged during learning. Odors activate unique yet overlapping ensembles 
of piriform neurons [34-36]. Therefore, silencing neurons that respond to one odor could partially interfere with the retrieval of information associated with other odors. To test this, we modified the behavioral protocol to separate Fos-tagging from fear conditioning. CFOS-tTA transgenic and wild-type control mice were injected with AAVtetO-hM4Di, and expression of hM4Di in piriform neurons was induced while mice were exposed to eugenol or beta-citronellol in a neutral environment, without subsequent foot shock. Mice were returned to doxycycline-containing diet and fearconditioned two days later to ethyl acetate (CS+). Behavioral testing of memory recall was performed one day later, after intraperitoneal injection of CNO (Figure 4A). Fostagging resulted in the labeling of sparse neural ensembles, similar in numbers to those tagged during olfactory fear conditioning $(1.1(0.3) \%$ of piriform neurons, $n=3$, Figure S3E and S4B). We found that learned escape behavior of cFos-tTA mice (experimental group, $\mathrm{n}=12$ ) was similar but somewhat attenuated compared to controls $(n=10)$. Both groups exhibited an escape behavior after CS+ presentation, indicated by the significant increase in the maximum velocity (WSRT, $W=55$, $p=0.0020$ and $W=54, p=0.0342$ for the control and experimental groups respectively; no difference in the maximum velocity ratio after/before between groups, MWT, $U=35$, $p=0.1072$, Figure $4 E, F$ and $\mathbf{G}$ ). The difference in the position of mice after $C S+$ and CS- presentation was also similar between groups (MWT, $U=37, p=0.1402$, Figure 4D). However, we observed that silencing neutral odor representations somewhat dampened the behavioral response to the $\mathrm{CS}_{+}$, as behavioral responses were less robust compared to controls when quantifying the position of mice in the box after $\mathrm{CS}+$ presentation (MWT, $\mathrm{U}=22, \mathrm{p}=0.0112$, Figure $4 \mathrm{~B}$ and $\mathrm{C}$ ). The attenuated behavioral responses we observe likely reflect the partial degradation of odor 
information as a result of the silencing of piriform neurons responsive to both the CS+ and the neutral odor.

\section{Silencing Fos-tagged piriform ensembles does not alter odor detection and discrimination}

Chemogenetic silencing of piriform ensembles Fos-tagged during olfactory fear conditioning could disrupt odor detection and discrimination rather than selectively affecting odor fear memory recall. To test this possibility, we monitored sniffing behavior of mice during an olfactory habituation assay (Figure 5A), a wellestablished test for odor detection and discrimination $[37,38]$. Fos-tagging of piriform neurons during olfactory fear conditioning was performed as described in Figure 3A. Three days later, changes in sniffing behavior in response to odor exposure were tested in a plethysmograph, while Fos-tagged neurons were silenced with CNO (see Figure S5C and Methods). We observed that mice of both experimental $(n=8)$ and control $(n=6)$ groups increased their sniff frequency upon the first presentation of pinene, a novel and neutral odor (1.6-fold increase relative to pre-odor baseline frequency; Figure 5B and D). Repeated exposure to the same odor (pinene or CS-) resulted in a decrease in the sniff frequency, reflecting habituation after 4 consecutive exposures (WSRT for the neutral odor, $\mathrm{W}=-21, \mathrm{p}=0.0312$ for the control and $W=-36, p=0.0078$ for the experimental group; Figure 5D). Subsequent presentation of the CS+ increased the sniff frequency (1.4-fold increase; WSRT, $\mathrm{W}=21, \mathrm{p}=0.0312$ for the control and $\mathrm{W}=34, \mathrm{p}=0.0156$ for the experimental group, Figure $5 \mathrm{E}$ ). Such changes in odor sampling behavior have been shown to report the detection and discrimination of different odor stimuli [37-39]. 
Furthermore, the baseline sniff frequency, the difference in sniff frequency between the first and fourth exposures of the same odor, and the difference in sniff frequency between the CS- after habituation and the CS+ were similar between control and experimental groups (MWTs, $U=12.5, p=0.1512$ for baseline; $U=22, p=0.8228$ for pin4-pin1 and $U=24, p>0.9999$ for $C S+-C S-4$, Figure 5C).

These data suggest that basic behaviors characteristic of odor sampling, detection and discrimination were unaffected by the silencing of piriform ensembles that were Fos-tagged during olfactory fear conditioning.

\section{Reactivation of Fos-tagged piriform ensembles mimics odor-evoked fear memory recall}

If piriform neural ensembles that were activated during olfactory fear conditioning encode an essential component of odor fear memories, then reactivation of these neurons may be sufficient to trigger memory recall.

To test this, we infected the PCx of cFos-tTA female mice with the activating DREADD AAV-tetO-hM3Dq (Figure S4D). We first trained mice to associate ethyl acetate with foot-shock, without CS- presentation. We then intraperitoneally injected CNO to reactivate hM3Dq-expressing piriform neurons that were Fos-tagged during learning (Figure 6A). Chemogenetics does not provide precise temporal control of neuronal activity, and CNO binding leads to a prolonged modulation of neuronal activation [40]. We therefore tested artificial memory retrieval in the open field box (see Figure $\mathbf{1 H}-\mathbf{K}$ for memory retrieval tested with prolonged odor exposure).

11.7 (1.7) \% of hM3Dq-expressing cells were reactivated upon CNO injection. As a point of comparison, 1.8 (1.4) \% of neurons were reactivated during olfactory memory retrieval (Supplementary Figure 6). CNO-mediated reactivation, similar to 
CS+ exposure, caused a significant decrease in exploratory behavior of fearconditioned mice $(n=7)$, compared to baseline exploratory behavior measured one day earlier (WSRT, $W=-26, p=0.0312$, Figure 6B). In contrast, reactivation of piriform neurons that were Fos-tagged during ethyl acetate exposure without foot shock $(n=7)$ did not affect exploratory behavior (WSRT, $W=-10, p=0.4688$, Figure 6B). Exploratory behavior after $\mathrm{CNO}$ injection normalized to baseline was significantly different between control and experimental groups (Mann Whitney test, $U=8$, $p=0.0379$, Figure $6 \mathrm{C}$ ). These data suggest that chemogenetic reactivation of piriform neurons that were active during odor-foot shock exposure is sufficient to trigger fear memory recall.

We next asked whether artificial memory recall depends on the specificity of the Fostagged neural ensemble: is piriform reactivation sufficient to trigger fear memory recall, as long as it includes the CS+-tagged ensemble? For this purpose, we generated a synthetic Fos-tagged ensemble, by alternating exposure to the CS+ odor paired with foot-shock with exposure to CS- neutral odors. We found that artificial reactivation of synthetic Fos-tagged ensemble did not result in changes in exploratory behavior indicative of memory recall (WSRT, $n=8, W=-4, p=0.8438$, Figure 6B). These data suggest that during artificial reactivation, PCx cannot extract meaningful information from the synthetic representation generated by the sequential presentation of CS+ and CS- odors. 


\section{Discussion}

The PCx has long been thought to provide the substrate for storing associative olfactory memories, yet the cellular substrates for olfactory learning and memory remain unknown. Using a cFos-dependent, intersectional genetic approach to visualize and manipulate piriform neurons activated during olfactory fear conditioning, we found that chemogenetic silencing of Fos-tagged ensembles robustly diminished a learned odor escape behavior without altering basic odor detection and discrimination. Furthermore, chemogenetic reactivation of Fos-tagged piriform ensembles resulted in reduced exploratory behavior in an open field assay, an effect that was similarly observed when exposing mice to the conditioned odor stimulus. Together, our experiments identify piriform neurons expressing Fos during learning as an essential neural circuit component for triggering odor fear memory recall.

\section{Localization of the odor fear memory trace}

The behavioral consequences of manipulating Fos-tagged piriform neurons suggest the formation of functional connections between piriform neurons and other association areas. Candidate target areas for the processing of odor-fear associations include the basolateral amygdala and the medial prefrontal cortex $[41,42]$, however, the relevant neural circuit components remain to be identified. We propose that odor fear memories are encoded in distributed ensembles of neurons throughout the brain, with different regions contributing to different components of the overall memory. The PCx could store information about odor objects, while associated emotions of past olfactory experiences could be stored in the amygdala (see $[2,47]$ for a similar model relative to contextual and auditory fear conditioning). Several studies have shown that PCx cells can encode information that carries 
behavioral significance $[21,33,44]$. Future studies investigating learning-induced changes in cellular and network properties of Fos-tagged neurons will establish if their silencing modifies the perception of the CS+, and thus the reactivation of the memory linked to the CS+, or if a memory trace of the odor-foot shock association is also formed within the Fos-tagged piriform ensembles.

\section{Technical limitations of Fos-tagging for studying odor fear memories}

In this study, we used two different fear conditioning paradigms, and two different retrieval contexts. It has previously been shown that the absence or presence of odorants not paired with foot shock (CS-) lead to a more or less generalized fear to odors similar to the reinforced conditioned stimulus (CS+) and to differential changes in the responses of piriform neurons [18]. Thus, PCx might be differentially involved in the retrieval of olfactory memories, depending on the presence or absence of CS-. In rats, PCx is not necessary three days after fear-conditioning to a CS+ odor without CS-, yet becomes indispensable when memory is tested one month after learning [21]. In our hands, Fos-tagged piriform ensembles are essential for odor fear memory retrieval three days after learning, when the CS+-shock presentation is coupled to the presentation of CS-.

A general limitation of the cFos-tTA system is that the tagging of neurons is transient. The duration of the expression of tTA-dependent proteins is determined by the time course of induction and the stability of cFos-tTA-dependent transcripts and proteins. Previous studies have shown that cFos-tTA-dependent expression of regulators of neural activity becomes undetectable by thirty days $[4,26,29]$. Therefore, the temporal involvement of PCx in memory retrieval cannot be addressed with Fostagging. 
Another constraint of the Fos-tagging system is its slow temporal dynamics. As a consequence, when training included the presentation of CS-, neurons responding to the CS- were tagged in addition to neurons responding to the CS+. We showed that the additional CS- "background" neurons had no significant impact on the outcome of the silencing experiment, as chemogenetic silencing of neurons activated during presentation of a CS- only moderately attenuated odor fear memory recall.

Interestingly, the number of Fos-expressing and Fos-tagged piriform neurons we observe is significantly lower than the number of odor-responsive neurons detected in electrophysiological recordings in awake mice $[34,45,46]$. However, in these experiments, a large fraction of cells exhibited low firing rates. Therefore, the Fostagged population could represent a subpopulation of neurons that is strongly activated by odor [47]. Fos-tagging could also mark plastic changes supporting memory formation $[48,49]$. Indeed, cFos mRNA levels decrease after injection of an antagonist of the NMDA receptor, a key player in the induction of synaptic plasticity [50], and long-term memory and synaptic plasticity are impaired when cFos production is perturbed in the central nervous system [51].

\section{Artificial reactivation of an olfactory memory trace}

Reactivation of neurons by CNO-mediated activation of DREADD receptors does not recapitulate the temporal characteristics of piriform odor responses and their modulation by active sampling $[34,45]$. Despite this, reactivation of piriform ensembles Fos-tagged during odor-foot shock pairing was sufficient to elicit a behavioral response. How piriform neural circuits and downstream target regions process such artificial activity patterns remains to be determined. One possibility is that despite the temporal limitations of memory trace reactivation, piriform network 
mechanisms can retrieve the perception of the reinforced conditioned stimulus. Consistent with this model, recent studies have shown that spatial patterns of odorevoked activity, in the absence of precise temporal information, are sufficient to decode odor identity $[34,35,45]$. Alternatively, it is possible that reactivation generates a state of fear, but without evoking the perception of the odor. Finally, reactivating a synthetic ensemble of neurons activated during exposure to both $\mathrm{CS}+$ and CS- stimuli did not elicit a measurable behavioral response. This observation suggests that behaviorally relevant information cannot be extracted from a neural ensemble representing conflicting (aversive versus neutral) information and further supports the specificity of the Fos-tagged ensemble. This result is consistent with the finding that the reactivation of an artificial contextual memory in the hippocampus competes with the retrieval of a learned context-shock association [52].

\section{Memory traces in hippocampus and PCx}

The hippocampus has been studied extensively for its role in spatial and contextual memory [53]. Recently, Fos-tagging of hippocampal neurons during contextual fear conditioning has provided important insight into the cellular and neural circuit mechanisms of learning and memory [4,23,52,54-56]. However, if principles of memory formation and storage in hippocampus-related neural networks apply to other cortical structures remains largely unknown. Interestingly, PCx and hippocampus share a similar circuit organization and both have been modeled as auto-associative networks [10]. In both regions, learning marks sparse and distributed ensembles of neurons that appear to lack topographic organization. Furthermore, hippocampal neurons tagged during contextual fear conditioning, and piriform neurons tagged during olfactory fear conditioning were both necessary and 
sufficient for memory retrieval $[4,6]$. Our findings thus reveal striking similarities between memory traces of contextual fear in the hippocampus and olfactory memory traces in the PCx. 


\section{Acknowledgements}

We thank Mark Mayford for sharing the cFos-tTA transgenic mouse line, and Brian Roth and Susumu Tonegawa for sharing plasmids. We thank Yves Dupraz and Gérard Parésys for their work on the behavioral apparatuses, Agatha Anet for help with behavioral experiments, Tristan Piolot for help with image acquisition, Philippe Mailly for help with cell quantification and Marion Ruinart de Brimont for help with cloning. We thank Karim Benchenane and Sophie Bagur for sharing their plethysmograph. We thank Rainer Friedrich, Anne Didier, Kevin Franks, Boris Gourévitch, Andreas Schaefer, and Claire Zhang for careful reading and critical comments on the manuscript. This work was supported by the "Amorçage de jeunes équipes" program (AJE201106) of the Fondation pour la Recherche Médicale (to A.F.), and a postdoctoral fellowship by the LabEx "MemoLife" (to C.M.B.).

\section{Author contributions}

Conceptualization, CMB and A.F.; Methodology, investigation and analysis, C.M.B, Y.D. (electrophysiology); Writing of original draft, C.M.B and A.F., with contributions from Y.D. and L.V.; Supervision and funding acquisition, C.M.B., L.V. and A.F.

\section{Declaration of Interests}

The authors declare no competing interests. 


\section{References}

1. Mouly, A.-M., and Sullivan, R. (2010). Memory and Plasticity in the Olfactory System: From Infancy to Adulthood. In The Neurobiology of Olfaction Frontiers in Neuroscience., A. Menini, ed. (Boca Raton (FL): CRC Press/Taylor \& Francis). Available at: http://www.ncbi.nlm.nih.gov/books/NBK55967/ [Accessed February 6, 2018].

2. Mayford, M., and Reijmers, L. (2015). Exploring Memory Representations with Activity-Based Genetics. Cold Spring Harb. Perspect. Biol. 8, a021832.

3. Tonegawa, S., Pignatelli, M., Roy, D.S., and Ryan, T.J. (2015). Memory engram storage and retrieval. Curr. Opin. Neurobiol. 35, 101-109.

4. Liu, X., Ramirez, S., Pang, P.T., Puryear, C.B., Govindarajan, A., Deisseroth, K., and Tonegawa, S. (2012). Optogenetic stimulation of a hippocampal engram activates fear memory recall. Nature $484,381-385$.

5. Reijmers, L.G., Perkins, B.L., Matsuo, N., and Mayford, M. (2007). Localization of a Stable Neural Correlate of Associative Memory. Science 317, 1230-1233.

6. Tanaka, K.Z., Pevzner, A., Hamidi, A.B., Nakazawa, Y., Graham, J., and Wiltgen, B.J. (2014). Cortical representations are reinstated by the hippocampus during memory retrieval. Neuron $84,347-354$.

7. Apicella, A., Yuan, Q., Scanziani, M., and Isaacson, J.S. (2010). Pyramidal cells in piriform cortex receive convergent input from distinct olfactory bulb glomeruli. J Neurosci 30, 14255-60.

8. Davison, I.G., and Ehlers, M.D. (2011). Neural circuit mechanisms for pattern detection and feature combination in olfactory cortex. Neuron 70, 82-94.

9. Wilson, D.A., and Sullivan, R.M. (2011). Cortical processing of odor objects. Neuron 72, 506-519. 
10. Haberly, L.B. (2001). Parallel-distributed processing in olfactory cortex: new insights from morphological and physiological analysis of neuronal circuitry. Chem Senses 26, 551-76.

11. Wilson, D.A., and Sullivan, R.M. (2011). Cortical processing of odor objects. Neuron 72, 506-519.

12. Johnson, D.M., Illig, K.R., Behan, M., and Haberly, L.B. (2000). New features of connectivity in piriform cortex visualized by intracellular injection of pyramidal cells suggest that "primary" olfactory cortex functions like "association" cortex in other sensory systems. J. Neurosci. Off. J. Soc. Neurosci. 20, 6974-6982.

13. Sadrian, B., and Wilson, D.A. (2015). Optogenetic Stimulation of Lateral Amygdala Input to Posterior Piriform Cortex Modulates Single-Unit and Ensemble Odor Processing. Front. Neural Circuits 9, 81.

14. Johenning, F.W., Beed, P.S., Trimbuch, T., Bendels, M.H.K., Winterer, J., and Schmitz, D. (2009). Dendritic compartment and neuronal output mode determine pathway-specific long-term potentiation in the piriform cortex. J. Neurosci. Off. J. Soc. Neurosci. 29, 13649-13661.

15. Kanter, E.D., and Haberly, L.B. (1990). NMDA-dependent induction of long-term potentiation in afferent and association fiber systems of piriform cortex in vitro. Brain Res. 525, 175-179.

16. Quinlan, E.M., Lebel, D., Brosh, I., and Barkai, E. (2004). A molecular mechanism for stabilization of learning-induced synaptic modifications. Neuron 41, 185-192.

17. Chapuis, J., and Wilson, D.A. (2011). Bidirectional plasticity of cortical pattern recognition and behavioral sensory acuity. Nat Neurosci 15, 155-61. 
18. Chen, C.-F.F., Barnes, D.C., and Wilson, D.A. (2011). Generalized vs. stimulusspecific learned fear differentially modifies stimulus encoding in primary sensory cortex of awake rats. J. Neurophysiol. 106, 3136-3144.

19. Sevelinges, Y., Gervais, R., Messaoudi, B., Granjon, L., and Mouly, A.-M. (2004). Olfactory fear conditioning induces field potential potentiation in rat olfactory cortex and amygdala. Learn. Mem. Cold Spring Harb. N 11, 761-769.

20. Shakhawat, A.M.D., Gheidi, A., MacIntyre, I.T., Walsh, M.L., Harley, C.W., and Yuan, Q. (2015). Arc-Expressing Neuronal Ensembles Supporting Pattern Separation Require Adrenergic Activity in Anterior Piriform Cortex: An Exploration of Neural Constraints on Learning. J. Neurosci. Off. J. Soc. Neurosci. 35, 1407014075 .

21. Sacco, T., and Sacchetti, B. (2010). Role of secondary sensory cortices in emotional memory storage and retrieval in rats. Science 329, 649-656.

22. Choi, G.B., Stettler, D.D., Kallman, B.R., Bhaskar, S.T., Fleischmann, A., and Axel, R. (2011). Driving opposing behaviors with ensembles of piriform neurons. Cell 146, 1004-1015.

23. Garner, A.R., Rowland, D.C., Hwang, S.Y., Baumgaertel, K., Roth, B.L., Kentros, C., and Mayford, M. (2012). Generation of a synthetic memory trace. Science 335, $1513-1516$.

24. Alexander, G.M., Rogan, S.C., Abbas, A.I., Armbruster, B.N., Pei, Y., Allen, J.A., Nonneman, R.J., Hartmann, J., Moy, S.S., Nicolelis, M.A., et al. (2009). Remote Control of Neuronal Activity in Transgenic Mice Expressing Evolved G ProteinCoupled Receptors. Neuron 63, 27-39.

25. Ferguson, S.M., Eskenazi, D., Ishikawa, M., Wanat, M.J., Phillips, P.E.M., Dong, Y., Roth, B.L., and Neumaier, J.F. (2011). Transient neuronal inhibition reveals 
opposing roles of indirect and direct pathways in sensitization. Nat. Neurosci. 14, 22-24.

26.Zhang, Z., Ferretti, V., Güntan, I., Moro, A., Steinberg, E.A., Ye, Z., Zecharia, A.Y., Yu, X., Vyssotski, A.L., Brickley, S.G., et al. (2015). Neuronal ensembles sufficient for recovery sleep and the sedative actions of $\alpha 2$ adrenergic agonists. Nat. Neurosci. $18,553-561$.

27. Adolphs, R. (2013). The biology of fear. Curr. Biol. CB 23, R79-93.

28. Gossen, M., Freundlieb, S., Bender, G., Muller, G., Hillen, W., and Bujard, H. (1995). Transcriptional activation by tetracyclines in mammalian cells. Science $268,1766-9$.

29. Cowansage, K.K., Shuman, T., Dillingham, B.C., Chang, A., Golshani, P., and Mayford, M. (2014). Direct reactivation of a coherent neocortical memory of context. Neuron $84,432-441$.

30. Datiche, F., Roullet, F., and Cattarelli, M. (2001). Expression of Fos in the piriform cortex after acquisition of olfactory learning: an immunohistochemical study in the rat. Brain Res. Bull. 55, 95-99.

31. Illig, K.R., and Haberly, L.B. (2003). Odor-evoked activity is spatially distributed in piriform cortex. J. Comp. Neurol. 457, 361-373.

32. Gomez, J.L., Bonaventura, J., Lesniak, W., Mathews, W.B., Sysa-Shah, P., Rodriguez, L.A., Ellis, R.J., Richie, C.T., Harvey, B.K., Dannals, R.F., et al. (2017). Chemogenetics revealed: DREADD occupancy and activation via converted clozapine. Science 357, 503-507.

33. Calu, D.J., Roesch, M.R., Stalnaker, T.A., and Schoenbaum, G. (2007). Associative encoding in posterior piriform cortex during odor discrimination and reversal learning. Cereb. Cortex N. Y. N 1991 17, 1342-1349. 
34. Bolding, K.A., and Franks, K.M. (2017). Complementary codes for odor identity and intensity in olfactory cortex. eLife 6 .

35. Roland, B., Deneux, T., Franks, K.M., Bathellier, B., and Fleischmann, A. (2017). Odor identity coding by distributed ensembles of neurons in the mouse olfactory cortex. eLife 6.

36.Stettler, D.D., and Axel, R. (2009). Representations of Odor in the Piriform Cortex. Neuron 63, 854-864.

37. Coronas-Samano, G., Ivanova, A.V., and Verhagen, J.V. (2016). The Habituation/Cross-Habituation Test Revisited: Guidance from Sniffing and Video Tracking. Neural Plast. 2016, 9131284.

38. Wesson, D.W., Donahou, T.N., Johnson, M.O., and Wachowiak, M. (2008). Sniffing behavior of mice during performance in odor-guided tasks. Chem. Senses 33, 581-596.

39. Verhagen, J.V., Wesson, D.W., Netoff, T.I., White, J.A., and Wachowiak, M. (2007). Sniffing controls an adaptive filter of sensory input to the olfactory bulb. Nat. Neurosci. 10, 631-639.

40. Alexander, G.M., Rogan, S.C., Abbas, A.I., Armbruster, B.N., Pei, Y., Allen, J.A., Nonneman, R.J., Hartmann, J., Moy, S.S., Nicolelis, M.A., et al. (2009). Remote control of neuronal activity in transgenic mice expressing evolved G proteincoupled receptors. Neuron 63, 27-39.

41. Dejean, C., Courtin, J., Karalis, N., Chaudun, F., Wurtz, H., Bienvenu, T.C.M., and Herry, C. (2016). Prefrontal neuronal assemblies temporally control fear behaviour. Nature 535, 420-424.

42. Gore, F., Schwartz, E.C., Brangers, B.C., Aladi, S., Stujenske, J.M., Likhtik, E., Russo, M.J., Gordon, J.A., Salzman, C.D., and Axel, R. (2015). Neural 
Representations of Unconditioned Stimuli in Basolateral Amygdala Mediate Innate and Learned Responses. Cell 162, 134-145.

43. Tonegawa, S., Liu, X., Ramirez, S., and Redondo, R. (2015). Memory Engram Cells Have Come of Age. Neuron 87, 918-931.

44.Mandairon, N., Kermen, F., Charpentier, C., Sacquet, J., Linster, C., and Didier, A. (2014). Context-driven activation of odor representations in the absence of olfactory stimuli in the olfactory bulb and piriform cortex. Front. Behav. Neurosci. 8, 138.

45. Miura, K., Mainen, Z.F., and Uchida, N. (2012). Odor representations in olfactory cortex: distributed rate coding and decorrelated population activity. Neuron 74, 1087-1098.

46. Zhan, C., and Luo, M. (2010). Diverse patterns of odor representation by neurons in the anterior piriform cortex of awake mice. J. Neurosci. Off. J. Soc. Neurosci. 30, 16662-16672.

47. Schoenenberger, P., Gerosa, D., and Oertner, T.G. (2009). Temporal control of immediate early gene induction by light. PloS One 4, e8185.

48. Cole, A.J., Saffen, D.W., Baraban, J.M., and Worley, P.F. (1989). Rapid increase of an immediate early gene messenger RNA in hippocampal neurons by synaptic NMDA receptor activation. Nature 340, 474-476.

49. Minatohara, K., Akiyoshi, M., and Okuno, H. (2015). Role of Immediate-Early Genes in Synaptic Plasticity and Neuronal Ensembles Underlying the Memory Trace. Front. Mol. Neurosci. 8, 78.

50. Tayler, K.K., Lowry, E., Tanaka, K., Levy, B., Reijmers, L., Mayford, M., and Wiltgen, B.J. (2011). Characterization of NMDAR-Independent Learning in the Hippocampus. Front. Behav. Neurosci. 5, 28. 
51. Fleischmann, A., Hvalby, O., Jensen, V., Strekalova, T., Zacher, C., Layer, L.E., Kvello, A., Reschke, M., Spanagel, R., Sprengel, R., et al. (2003). Impaired longterm memory and NR2A-type NMDA receptor-dependent synaptic plasticity in mice lacking c-Fos in the CNS. J. Neurosci. Off. J. Soc. Neurosci. 23, 9116-9122.

52. Ramirez, S., Liu, X., Lin, P.-A., Suh, J., Pignatelli, M., Redondo, R.L., Ryan, T.J., and Tonegawa, S. (2013). Creating a false memory in the hippocampus. Science $341,387-391$.

53. Basu, J., and Siegelbaum, S.A. (2015). The Corticohippocampal Circuit, Synaptic Plasticity, and Memory. Cold Spring Harb. Perspect. Biol. 7.

54. Cai, D.J., Aharoni, D., Shuman, T., Shobe, J., Biane, J., Song, W., Wei, B., Veshkini, M., La-Vu, M., Lou, J., et al. (2016). A shared neural ensemble links distinct contextual memories encoded close in time. Nature 534, 115-118.

55. Roy, D.S., Kitamura, T., Okuyama, T., Ogawa, S.K., Sun, C., Obata, Y., Yoshiki, A., and Tonegawa, S. (2017). Distinct Neural Circuits for the Formation and Retrieval of Episodic Memories. Cell 170, 1000-1012.e19.

56. Ryan, T.J., Roy, D.S., Pignatelli, M., Arons, A., and Tonegawa, S. (2015). Memory. Engram cells retain memory under retrograde amnesia. Science 348, 1007-1013.

57. Meziane, H., Ouagazzal, A.-M., Aubert, L., Wietrzych, M., and Krezel, W. (2007). Estrous cycle effects on behavior of C57BL/6J and BALB/cByJ female mice: implications for phenotyping strategies. Genes Brain Behav. 6, 192-200.

58. Padilla, S.L., Quu, J., Nestor, C.C., Zhang, C., Smith, A.W., Whiddon, B.B., Rønnekleiv, O.K., Kelly, M.J., and Palmiter, R.D. (2017). AgRP to Kiss1 neuron signaling links nutritional state and fertility. Proc. Natl. Acad. Sci. U. S. A. 114, 2413-2418. 
59. Srinivasan, S., and Stevens, C.F. (2017). A quantitative description of the mouse piriform cortex.

60. Suzuki, N., and Bekkers, J.M. (2006). Neural Coding by Two Classes of Principal Cells in the Mouse Piriform Cortex. J. Neurosci. 26, 11938-11947. 


\section{Figure legends}

Figure 1. Behavioral expression of odor fear memory depends on the retrieval context

(A) During fear conditioning, mice are trained to associate ethyl acetate (CS+) with foot shock. The CS+ is presented at one end of a rectangular training box, and the corresponding half-side of the box is electrified. Training includes the presentation of two other odors that are not paired with foot shock (CS-, eugenol and beta-citronellol). The CS+ is presented 4 times (2 times on each side) and each CS- 3 times, for $7 \mathrm{~s}$ each. The order and side of odor presentations is pseudo-randomized (see Figure S1). Memory retrieval is tested three days later, either in a rectangular testing box or in an open field, with very short or prolonged odor exposure respectively. In the open field, baseline exploratory activity in the absence of odor (air) is measured one day earlier.

(B-J) Wild-type mice are used for testing odor-evoked fear memory recall in the open field, and cFos-tTA mice injected with AAV-GCaMP are used for testing in the rectangular testing box.

(B-G) Memory retrieval in a box having the same shape and dimensions as the training box.

(B) In the rectangular testing box, the $\mathrm{CS}_{+}$is presented 4 times (2 times on each side), and each CS- is presented once on each side, for $7 \mathrm{~s}$ each. The testing session is video-recorded and the position of the mouse's centroid in the box along the x-axis is extracted. The length of the box is normalized to 1 . Since the odor is presented randomly on the left or right side of the testing box, 0 is defined as the extremity where the odor is presented. For velocity measurements, a $7 \mathrm{~s}$ time window is defined both before and after stimulus onset. 
(C) Averaged position of mice as a function of time after CS+ (black dashed line) and CS- (blue line) presentation.

(D-E) 3 additional mice (etac control) did not receive foot shock after ethyl-acetate presentation.

(D) Position in the testing box $7 \mathrm{~s}$ after $\mathrm{CS}_{+}, \mathrm{CS}-$, or etac presentation. Mice are further away from the odor port after CS+ presentation than after CS- presentation $(n=9, W=45, p=0039)$.

(E) Maximum velocity $7 \mathrm{~s}$ before and $7 \mathrm{~s}$ after CS+ (left), CS- (middle) or etac (right) presentation. After CS+ presentation, the maximum velocity significantly increases $(n=9, W=43, p=0.0078)$.

(F) Position $7 \mathrm{~s}$ after CS- presentation subtracted from the position $7 \mathrm{~s}$ after CS+ presentation.

(G) Maximum velocity ratio after/before CS- subtracted from the maximum velocity ratio after/before CS+ presentation.

$(\mathrm{H}-\mathrm{K})$ Memory retrieval in an open field. Ethyl acetate is presented above the open field from minutes 15 to 18 (testing). To measure baseline exploratory activity, air is diffused above the open field the day before (baseline). Ethyl acetate exposure lead to a decrease in exploratory behavior in mice previously fear-conditioned to ethyl acetate ( $n=6$, in red). Previous exposure to ethyl acetate without foot shock does not alter exploratory behavior upon re-exposure to ethyl acetate ( $n=5$, in black).

$(\mathrm{H}, \mathrm{I})$ Training includes the presentation of two other odors that are not paired with foot shock (CS-).

(H) Average speed in $\mathrm{cm} / \mathrm{min}$. As a measure for exploratory activity, the distance travelled is computed from minute 15 to 18 (corresponding to odor exposure during testing). 
(I) Average speed ratio testing/baseline.

$(\mathrm{J}, \mathrm{K})$ Experimental design: same as in $(\mathrm{H}, \mathrm{I})$, except that training does not include the presentation of CS-.

(J) Average speed in $\mathrm{cm} / \mathrm{min}$.

(K) Average speed ratio testing/baseline.

Dots represent individual mice, averaged data are shown as median and interquartile range. ${ }^{* *} p<0.01 ;{ }^{*} p<0.05 ;$ n.s. not significant. 


\section{Figure 2. Fos-expressing piriform neurons can be tagged and functionally}

manipulated.

(A-D) Scheme of genetic strategy and histological characterization of Fos-tagged piriform ensembles.

(A) The "silencing" and "activating" TetTag system. AAVs (red) expressing DREADDs under the control of the tetO promoter were injected in the piriform cortex of cFos-tTA transgenic mice (see also Figure S3). hM4Di or hM3Dq are expressed in active Fos-expressing piriform neurons upon doxycycline removal.

(B-D) Mice were fear conditioned to ethyl acetate five days after doxycycline removal. Training includes the presentation of two other odors that are not paired with foot shock (CS-, eugenol and beta-citronellol). Mice were perfused one hour after the start of the experiment (B) or three days later (C-D). (B) Endogenous Fos expression. (C) Neurons expressing hM4Di were visualized using anti-HA immunohistochemistry. (D) Neurons expressing hM3Dq were visualized with mCherry fluorescence. Neurotrace counterstain in blue. Scale bars: $50 \mu \mathrm{m}$.

The number below the coronal sections indicate the median percentage (interquartile range) of tagged neurons quantified in 2 or 3 mice.

(E-I) Electrophysiological characterization of Fos-tagged piriform neurons.

(E) Resting membrane potential in $\mathrm{mV}$, before (baseline, $\mathrm{BL}$ ) and after $\mathrm{CNO}$ application. The resting membrane potential does not differ between DREADDexpressing and non-expressing cells (Ctrl). After CNO application, it does not change in non-expressing cells (Ctrl), while hM4Di-expressing cells hyperpolarize and hM3Dq-expressing cells depolarize. 
(F) The normalized membrane potential in $\%$ to baseline in different cell types after CNO application. Pyramidal-like neurons (PN) are marked as circles, semilunar-like cells (SL) as triangles and fast-spiking interneurons (FS) as squares (see Figure S2). (G-I) The excitability of DREADD-expressing cells is measured as the number of action potentials (APs) resulting from the third depolarizing current step, counting from the first step that elicits spiking (steps of $20 \mathrm{pA}$ ).

(G) Representative traces before (baseline, BL) and after CNO application.

(H) Number of APs before (baseline, BL) and after CNO application. After CNO application, the number of APs in DREADD-negative cells (Ctrl) does not change, significantly decreases in hM4Di-expressing cells and significantly increases in hM3Dq-expressing cells.

(I) Normalized number of APs in \% to baseline in different cell types after CNO application. Cell types are marked as in $(F)$.

Data are shown as individual data points, median and interquartile range, ${ }^{* *} p<0.01$. 


\section{Figure 3. Silencing Fos-tagged piriform ensembles impairs odor fear memory}

recall.

(A) Experimental design: cFos-tTA transgenic mice (experimental group, $n=8$ ) or wild-type mice (control group, $n=8$ ) are injected with AAV-tetO-hM4Di in both hemispheres of piriform cortex. Ten days later, mice are habituated to the training and testing boxes. Upon doxycycline (DOX) removal, mice are fear-conditioned, and hM4Di expression (green circles) is induced in Fos-tagged neurons (filled in blue and yellow, see Figure S4). Mice are returned to DOX-containing diet to avoid further expression of hM4Di. Three days later, all mice are intraperitoneally injected with CNO, which silences hM4Di-expressing neurons (black crosses), and memory retrieval is tested in the rectangular testing box (Figure 1B).

( $\mathrm{B}$ to $\mathrm{G}$ ) Olfactory memory retrieval after $\mathrm{CNO}$ injection, assessed by the position of mice in the testing box and their maximum velocity upon odor exposure. Memory retrieval was significantly impaired in cFos-tTA mice in which piriform neurons that were active during learning were chemogenetically silenced during memory recall (experimental group). See Figure S5 for unilaterally infected mice.

(B) Averaged position of mice in the testing box as a function of time after CS+ (black dashed line) and CS- (blue line) presentation.

(C-G) Control group: color-coded black; experimental group: color-coded green.

(C) Position in the testing box $7 \mathrm{~s}$ after CS+ or CS- presentation.

(D) Position $7 \mathrm{~s}$ after CS- presentation subtracted from the position $7 \mathrm{~s}$ after CS+ presentation.

(E) Maximum velocity during a $7 \mathrm{~s}$ time window before and after $\mathrm{CS}+$ presentation.

(F) Maximum velocity during a $7 \mathrm{~s}$ time window before and after CS- presentation.

(G) Maximum velocity ratio after/before CS- subtracted from the maximum velocity 
ratio after/before CS+ presentation.

Dots represent individual mice, and averaged data are shown as median and interquartile range, ${ }^{*} p<0.05 ;{ }^{* *} p<0.01 ;$ n.s. not significant. 
Figure 4. Memory impairments are specific to the piriform ensemble that is Fos-tagged during learning

(A) Experimental design: dissociating Fos-tagging and fear conditioning. Upon doxycycline (DOX) removal, mice are presented with an odor in a neutral environment (C: eugenol, eug. or beta-citronellol, beta-cit.). hM4Di expression (green circles) is induced in Fos-tagged neurons (filled in orange, also see Figure S3 and S4). Two days later, mice are fear conditioned to ethyl-acetate (CS-: limonene and beta-cit. when Fos-tagged with eug., limonene and eug. when Fos-tagged with betacit.). One day later, memory retrieval is tested in the presence of CNO, which silences hM4Di-expressing neurons (black crosses).

(B-G) Olfactory memory retrieval after CNO injection, assessed by the position of mice in the testing box and their maximum velocity. Memory retrieval was moderately attenuated in cFos-tTA mice expressing hM4Di in neurons that were Fos-tagged during presentation of a neutral odor (experimental group, $n=12$ ), compared to WT mice (control group, $n=10$ ).

(B) Averaged position of mice as a function of time after CS+ and CS- presentation.

(C) Position of mice in the testing box $7 \mathrm{~s}$ after CS+ or CS- presentation.

(D) Position $7 \mathrm{~s}$ after CS- presentation subtracted to the position $7 \mathrm{~s}$ after CS+ presentation.

(E) Maximum velocity during the $7 \mathrm{~s}$ before and after CS+ presentation.

(F) Maximum velocity during a $7 \mathrm{~s}$ time window before and after CS- presentation.

(G) Maximum velocity ratio after/before CS- subtracted from the maximum velocity ratio after/before CS+ presentation.

Dots represent individual mice, and averaged data are shown as median and interquartile range, ${ }^{*} p<0.05 ;{ }^{* *} p<0.01 ;$ n.s. not significant. 


\section{Figure 5. Silencing Fos-tagged piriform ensembles does not interfere with odor}

detection and discrimination.

(A) (Top) Experimental design: Fos-tagging during olfactory fear conditioning is performed as described in Figure 2 (see also Figure S4). Three days after fear conditioning, sniffing behavior is quantified in a plethysmograph. Mice are intraperitoneally injected with $\mathrm{CNO}$, habituated to the plethysmograph for $15 \mathrm{~min}$, and each odor is then presented for $8 \mathrm{~s}$ with a 2.5 min inter-trial interval. pin: pinene, CS-: beta-citronellol, CS+: ethyl-acetate.

(Bottom) Representative sniff recordings from one mouse when exposed to pinene (1st presentation) or the CS+ (ethyl-acetate).

(B-C) Dots represent individual mice, and averaged data are shown as median and interquartile range.

(D-E) Each thin line represents data of individual mice, the squares and thick lines represent averaged data (median and interquartile range).

(B) Baseline sniff frequency, corresponding to the averaged sniff frequency $8 \mathrm{~s}$ before odor onset.

(C-E) For both the control $(n=6)$ and experimental group $(n=8)$, repeated exposure to the same odor resulted in a decrease in the sniff frequency (habituation), while presentation of a different odor resulted in an increase in the sniff frequency.

(C) The changes in sniff frequency after presentation of the same or a different odor are similar between the experimental and the control group. The sniff frequency during the fourth presentation of pinene is subtracted from its first presentation (pin4pin1), or the sniff frequency during the fourth presentation of the CS- is subtracted from the sniff frequency during the CS+ presentation (CS+-CS-4). 
(D) Sniff frequency for the first to fourth presentation of pinene: control (left) and experimental group (right).

(E) Sniff frequency for the first to fourth presentation of the CS-, and subsequent presentation of the CS+: control (left) and experimental group (right).

${ }^{*} p<0.05 ;{ }^{* *} p<0.01 ;$ n.s.: not significant. 


\section{Figure 6. Reactivation of Fos-tagged piriform ensembles mimicks odor-evoked}

fear memory recall.

(A) Experimental design: artificial odor fear memory recall tested in an open field assay (see Figure 1). cFos-tTA transgenic mice are injected with AAV-tetO-hM3Dq in piriform cortex to induce hM3Dq expression in Fos-expressing neurons during training (see also Figure S3 and S4). Mice are trained to associate ethyl-acetate with foot shock (CS+), and mice exposed to ethyl-acetate without foot shock serve as controls. An additional group is trained to associate ethyl-acetate with foot shock and training includes the presentation of two CS- (eugenol and beta-citronellol). Two days later, baseline exploratory activity in an open field is measured during $22 \mathrm{~min}, 5 \mathrm{~min}$ after i.p. injection of saline (test 1). The next day (test 2), CNO is i.p injected to reactivate Fos-tagged, hM3Dq-expressing piriform neurons (see Figure S6) and activity of mice in the open field is measured again.

(B) Average speed in $\mathrm{cm} / \mathrm{min}$ : distance travelled during the entire testing session divided by the duration of the session. CNO injection leads to a decrease in exploratory behavior in mice previously fear conditioned to ethyl acetate in the absence of CS- $(n=7)$. Previous exposure to ethyl acetate without foot shock does not alter exploratory behavior upon re-activation of Fos-tagged piriform ensembles $(n=7)$.

Reactivation of piriform ensembles Fos-tagged during olfactory fear conditioning including reinforced (CS+) and non-reinforced (CS-) odor stimuli does not affect exploratory behavior $(\mathrm{n}=8)$.

(C) Average speed ratio testing/baseline.

Dots represent individual mice, and averaged data are presented as median and interquartile range. ${ }^{*} p<0.05$; n.s. not significant. 


\section{STAR METHODS}

\section{CONTACT FOR REAGENT AND RESOURCE SHARING}

Further information and requests for resources and reagents should be directed to and will be fulfilled by the Lead Contact, Alexander Fleischmann (alexander fleischmann@brown.edu).

\section{EXPERIMENTAL MODEL AND SUBJECT DETAILS}

Mice were housed at $24^{\circ} \mathrm{C}$ with a 12 -hour light/12-hour dark cycle with standard food and water provided ad libitum. Mice were group-housed with littermates until the beginning of surgery and then single-housed in ventilated cages throughout the duration of the experiment. CFos-tTA mice [5] were originally generated on a mixed $\mathrm{C} 57 \mathrm{BL} / 6 \times \mathrm{DBA} / 2$ genetic background and backcrossed for at least 6 generations into a pure C57BL/6N genetic background (Charles River). Wild-type control animals $(\mathrm{n}=46)$ were siblings that did not carry the cFos-tTA transgene. The age of mice at the time of behavioral testing ranged from 10 to 15 weeks. Male mice $(n=48)$ were used for the hM4Di-mediated neural silencing, and littermate females $(n=40)$ for the hM3Dq-mediated neural activation experiments. No differences in open field performance depending on the phase of the estrous cycle were found in female mice [57], and CNO injection does not disrupt the estrous cycle [58]. Mice were fed a diet containing $1 \mathrm{~g} / \mathrm{kg}$ doxycycline (SSNIFF) for a minimum of 4 days before surgery. Experiments were carried out according to European and French national institutional animal care guidelines (protocol APAFIS\#2016012909576100).

\section{METHOD DETAILS}




\section{Constructs and viruses}

The hSynapsin promoter of pAAV-hSyn-HA-hM4Di-IRES-mCitrine (kindly provided by Dr. B. Roth, University of North Carolina at Chapel Hill) was excised using the restriction enzyme Xbal (New England Biolabs) and replaced by the Tetracycline Response Element of a pAAV-TRE-EYFP (excised with Xba1 et Nhe1, plasmid kindly provided by Dr. S. Tonegawa, MIT, Cambridge). The pAAV-pTRE-tighthM3Dq-mCherry [26] was purchased from Addgene (\#66795). Adeno-associated viruses (AAVs) were generated at Penn Vector Core, University of Pennsylvania (serotype $8,10^{13}$ genome copies/mL, 1:2 dilution with sterile PBS on the day of injection).

\section{Stereotaxic injection}

Mice were anaesthetized with ketamine/xylazine $(100 \mathrm{mg} / \mathrm{kg} / 10 \mathrm{mg} / \mathrm{kg}$, SigmaAldrich) and $A A V$ vectors were injected stereotaxically into the piriform cortex (PCx, coordinates relative to bregma: anterior-posterior, $-0.6 \mathrm{~mm}$; dorsal-ventral, $-4.05 \mathrm{~mm}$; lateral, $4.05 \mathrm{~mm}$ and $-4.05 \mathrm{~mm}$ ). Using a micromanipulator and injection assembly kit (Narishige; WPI), a pulled glass micropipette (Dutscher, 075054) was slowly lowered into the brain and left for 30 seconds in place before infusion of the virus at an injection rate of $0.2-0.3 \mu \mathrm{L}$ per min. $0.8-1 \mu \mathrm{L}$ of virus was sufficient to infect a large area of PCx. The micropipette was left in place for an additional 3-4 min and then slowly withdrawn to minimize diffusion along the injector tract.

\section{Immunohistochemistry}

Mice were deeply anesthetized with pentobarbital and perfused transcardially with phosphate-buffered saline (PBS) followed by $4 \%$ paraformaldehyde. Brains were 
post-fixed $4 \mathrm{~h}$ in $4 \%$ paraformaldehyde, and $100-200 \mu \mathrm{m}$ coronal sections were cut with a vibrating blade microtome (Microm Microtech). Sections were permeabilized in $0.1 \%$ Triton-X100/PBS (PBST) for $1 \mathrm{~h}$, then blocked in $2 \%$ heat-inactivated horse serum (HIHS)-PBST for $1 \mathrm{~h}$. Sections were incubated in 2\% HIHS-PBST containing polyclonal primary antibodies (rabbit anti-HA, 1:200, Cell Signaling; rabbit anti-GFP, 1:1000, Invitrogen; goat anti-cFos, 1:500, Santa Cruz) with gentle agitation at $4^{\circ} \mathrm{C}$ overnight. Next, sections were rinsed 3 times in PBST for 20 min, blocked in $2 \%$ HIHS-PBST for $1 \mathrm{~h}$, and incubated in NeuroTrace 640/660 (Invitrogen) and speciesappropriate Alexa Fluor 488 (green) and Alexa Fluor 568 (red)-conjugated secondary antibodies $\left(1: 1000\right.$, Invitrogen) at $4^{\circ} \mathrm{C}$ overnight. Sections were washed 2 times in PBST and 1 time in PBS, mounted on slides and coverslipped with Vectashield mounting medium (Vectorlabs). Images were acquired as Z-stacks (70 to $140 \mu \mathrm{m}$ in total thickness, step size $7 \mu \mathrm{m}$ ) with a Leica SP5 confocal microscope or as single plane sections with a Zeiss Axio Zoom microscope and processed in Fiji.

The numbers of cells were calculated as numbers of cells per cubic millimeter. An estimate of the total number of neurons (counterstained with NeuroTrace) per cubic millimeter $\left(7.1 \mathrm{e} 4 \mathrm{~mm}^{-3}\right)$ was obtained by counting neurons in representative volumes containing the three layers of PCx. Numbers are consistent with those obtained by [59].

\section{Electrophysiology}

Parasagittal or coronal slices (300 $\mu \mathrm{m}$ thick) of PCx were prepared from 6-8 week-old cFos-tTA mice injected with AAV-tetO-HA:hM4Di-IRES-mCitrine or AAV-tetOhM3Dq:mCherry. Three days after olfactory fear conditioning, animals were anaesthetized with ketamine and xylazine $(100 \mathrm{mg} / \mathrm{kg} / 10 \mathrm{mg} / \mathrm{kg}$, Sigma-Aldrich), 
perfused with ice-cold ACSF (125 mM NaCl, $2.5 \mathrm{mM} \mathrm{KCl}, 25 \mathrm{mM}$ glucose $25 \mathrm{mM}$ $\mathrm{NaHCO}_{3}, 1.25 \mathrm{mM} \mathrm{NaH}_{2} \mathrm{PO}_{4}, 2 \mathrm{mM} \mathrm{CaCl}, 1 \mathrm{mM} \mathrm{MgCl} 2,1 \mathrm{mM}$ pyruvic acid, bubbled with $95 \% \mathrm{O}_{2}$ and $5 \% \mathrm{CO}_{2}$ and adjusted to $295 \pm 5$ mOsm osmolarity), and decapitated. The brain was cooled with ice-cold ACSF solution and then sliced using a 7000SM2 vibrating microtome (Campden Instruments, UK). Slices were incubated in the same solution at $34^{\circ} \mathrm{C}$ for one hour and then continuously perfused with ACSF solution (2 $\mathrm{mL} / \mathrm{min}$ ) at $34^{\circ} \mathrm{C}$ in the recording chamber. DREADD expression was detected with two-photon excitation (830nm, ChameleonMRU-X1, Coherent, UK) under a Scientifica TriM Scope II microscope (LaVision, Germany), with a 60x waterimmersion objective. Whole-cell recording pipettes with 5-7 $\mathrm{M} \Omega$ resistance were filled with the following solution (in $\mathrm{mM}$ ): 122 Kgluconate, $13 \mathrm{KCl}, 10$ HEPES, 10 phosphocreatine, 4 Mg-ATP, 0.3 Na-GTP, 0.3 EGTA (adjusted to pH 7.35 with KOH). The recording solution also contained the morphological tracer Alexa Fluor $594(5 \mu \mathrm{M}$, red channel) for non-expressing and hM4Di-positive cells or the $\mathrm{Ca}^{2+}$-sensitive dye Fura-2 (300 $\mu \mathrm{M}$, replacing EGTA in the recording solution, green channel) for hM3Dq-positive cells, to enable identification of the patched cell and to visualize its morphology. The excitability of cells was measured in current-clamp mode by $500 \mathrm{~ms}$ steps of current injections from -300 to +500 pA with steps of $20 \mathrm{pA}$. We compared the number of action potentials triggered by equivalent depolarization at each step 60 pA above the action potential threshold before and after CNO $(5 \mu \mathrm{M})$ application. The series resistance was usually $<20 \mathrm{M} \Omega$, and data were discarded if it changed by more than $20 \%$ during the recording. Signals were amplified using EPC10-2 amplifiers (HEKA Elektronik, Lambrecht, Germany). Voltage-clamp recordings were filtered at $5 \mathrm{kHz}$ and sampled at $10 \mathrm{kHz}$, and current-clamp recordings were filtered at $10 \mathrm{kHz}$ and sampled at $20 \mathrm{kHz}$, with the Patchmaster v2×32 program (HEKA 


\section{Elektronik).}

\section{Behavioral apparatus}

A training box was used to train mice to escape from an odor. The box was rectangular ( $\mathrm{L} 57 \mathrm{~cm}, \mathrm{~W} 17 \mathrm{~cm}, \mathrm{H} 64 \mathrm{~cm}$ ), with a grid floor made of 72 stainless-steel rods (diameter $=6 \mathrm{~mm}$, space between rods $=2 \mathrm{~mm}$ ) and walls made of gray Altuglas Visio. Current was delivered by an aversive stimulator (MedAssociates, 115V, $60 \mathrm{~Hz}$ ). A custom-made switcher allowed an electric foot shock $(0.6 \mathrm{~mA}, 0.6 \mathrm{~ms})$ to be applied independently to either half of the box. A testing box was used to test memory retrieval. Its dimensions were the same as the training box. Materials for the walls (black expanded PVC) and floor (white Altuglas) were different from the training box to create a different context. Odor ports were located at each extremity of the training and testing boxes.

The open field box consisted of a white Plexiglas (6 mm thickness) container $(50 \mathrm{~cm}$ x $50 \mathrm{~cm} \times 38$ height).

Sniffing behavior was monitored in freely moving mice using a plethysmograph (Emka Technologies). Electrical signals were amplified using RHD 2132 amplifier boards (Intan Technologies) and band-pass filtered (1-30 Hz).

Odors were delivered using an 8 channels olfactometer (Automate Scientific). The olfactometer output was split into two channels downstream of the odor bottles, and controlled by independent valve controllers for left/right presentation. A continuous clean air stream and the output from the olfactometer valves converged into a custom-made manifold. To avoid pressure changes when presenting the odors, a valve connected to a bottle of mineral oil (Sigma-Aldrich) was opened when the valves connected to the odor bottles were closed. The following odors (Sigma- 
Aldrich), at a concentration of $1 \%(\mathrm{vol} / \mathrm{vol})$ in mineral oil were used: ethyl acetate, beta-citronellol, eugenol, limonene, and pinene.

\section{Behavioral procedures}

AAVs were stereotaxically injected in both hemispheres of PCx of cFos-tTA transgenic mice and littermate wild-type controls. Mice were given 10 days to recover, before being habituated on day 11 to the training and testing/open field/plethysmograph boxes (40 and $10 \mathrm{~min}$ each). 24 hours after habituation, doxycycline was removed and replaced by a regular diet. Five days later, mice were fear conditioned to ethyl acetate or exposed to an odor (odor "C") in a neutral environment. Immediately after fear conditioning or odor presentation, mice were put back on a diet containing doxycycline. During fear conditioning, mice were freely moving around the conditioning box. Ethyl acetate (the CS+) was presented when the mouse was located at one end of the box, and the corresponding half-side of the box' floor was electrified for $0.6 \mathrm{~s}(0.6 \mathrm{~mA})$. The foot shock was delivered 4 seconds after $\mathrm{CS}+$ presentation, and the CS+ lasted for an additional 2 seconds. Mice learned to escape to the opposite side when the CS+ was presented. The CS+ was presented 4 times, 2 times on each side of the box. When fear conditioning included presentation of two non-reinforced conditioned stimuli (CS-1 and CS-2), the choice between CS+ and CS- as well as the side of presentation was pseudo-randomized. Training always started with one presentation of each CS- followed by the CS+. The two CS- odors were presented during 7 seconds, 3 times each, with a total presentation of 3 on the right side and 3 on the left side. The mean time interval between two odor presentations was 3 minutes.

In the rectangular testing box, the CS+ was presented 4 times ( 2 times on each side), 
and each CS- was presented once on each side, for $7 \mathrm{~s}$ each (mean time interval between two odors: 3 minutes). As for the training session, the order and side of odor presentations was pseudo-randomized: testing always started with one presentation of each CS-, followed by one CS+ presentation. In the open field, the olfactometer outputs were placed above the open field box. Mice were placed in the middle of the box at the start of each session.

\section{Drugs}

Clozapine-N-oxide (C0832, Sigma-Aldrich) was first dissolved in dimethylsulfoxide (DMSO, D2438, Sigma, final concentration of $1 \% \mathrm{vol} / \mathrm{vol}$ ), then further diluted in $0.9 \%$ sterile saline solution to a final concentration of $0.2 \mathrm{mg} / \mathrm{mL}$. Aliquots were stored for up to two months at $-20^{\circ} \mathrm{C}$ and equilibrated to room temperature before injection. The solution was administered intraperitoneally $(3 \mathrm{mg} / \mathrm{kg})$. After injection, mice were left undisturbed for 25 minutes (Figure 2 and 4) or 5 minutes (Figure 3 and 5) in their home cage before the start of the experiment.

\section{QUANTIFICATION AND STATISTICAL ANALYSIS}

\section{Cell counting}

Z-stacks (7 $\mu \mathrm{m}$ step) were acquired with a Leica SP5 confocal microscope with a 20X

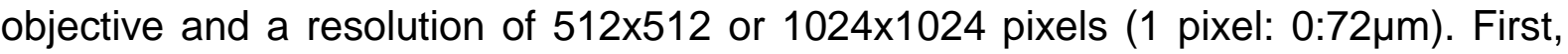
the PCx was delineated on a maximum projection of each stack using a customwritten ImageJ macro. For each mouse, 2 to 4 sections (median volume 9.3e-2 (9.4e3) $\mathrm{mm}^{3}$ ) were analyzed, from one or both hemispheres, at $Y=-0.8$ and $Y=-1.6 \mathrm{~mm}$ relative to bregma. 
HA-stained neurons (hM4Di) were counted by hand. hM3Dq-mCherry and Fospositive neurons were counted using a custom-written ImageJ plugin. After preprocessing the image (Subtract Background, Remove Outliers and Median Filter), the z-stack is thresholded using the RenyiEntropy algorithm. On each slice of the stack, objects with an area smaller than $40 \mu \mathrm{m}^{2}$ are removed using the Analyze Particle command in ImageJ. A mask of each slice from the stack is created containing the filled outline of the measured particles. The number of cells is the number of $3 D$ objects detected in the stack. Automated cell detection can be prone to errors; thus, the detection efficiency was checked on a few pictures by two people blind to the experimental conditions. An estimate $\mathrm{E}_{\mathrm{TOT}}$ of the total number of neurons (counterstained with NeuroTrace) per cubic millimeter $\left(7.1 \mathrm{e} 4 \mathrm{~mm}^{-3}\right.$ ) was obtained by counting neurons in representative volumes containing the three layers of PCx. Numbers are consistent with those obtained by [59]. The total number of neurons in each stack was calculated by multiplying $\mathrm{E}_{\mathrm{TOT}}$ by the volume of the stack.

\section{Electrophysiology}

Electrophysiological data were analyzed with custom-made software in Python 3.0 and averaged in MS Excel (Microsoft, USA). Cell types were classified based on their excitability, input resistance, the resting membrane potential, as described in [60] and morphology obtained from 2-photon images

\section{Behavioral data}

Behavioral sessions were video recorded and analyzed using custom-written Matlab programs (https://git.io/fxVeE). Parameters extracted from multiple CS+ (CS-) presentations were averaged for each mouse, when testing occurred in the 
rectangular testing box (Figure 1, 3 and 4).

\section{Statistics}

Statistical analysis was performed with Prism (GraphPad). Non-parametric tests were used: Mann-Whitney test (MWT) for between group comparison, and Wilcoxon matched-pairs signed rank test (WSRT) for within group comparison. Values are represented as median (interquartile range) unless otherwise stated. The $n$ represents number of mice, or number of recorded neurons for the electrophysiology experiment.

\section{DATA AND SOFTWARE AVAILABILITY}

Data are available upon requests. 


\section{A Training}

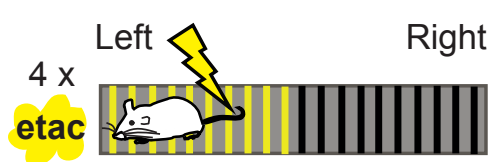

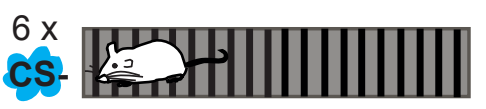

pseudo-random order CS+/CS- and Left/Right
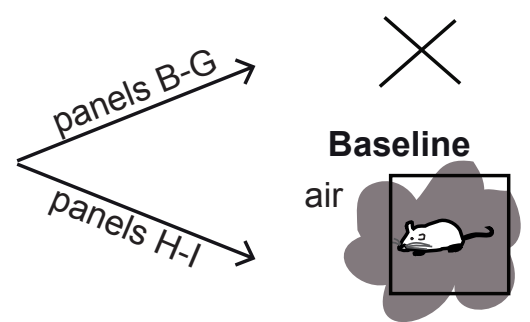

Testing

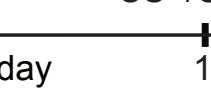

B
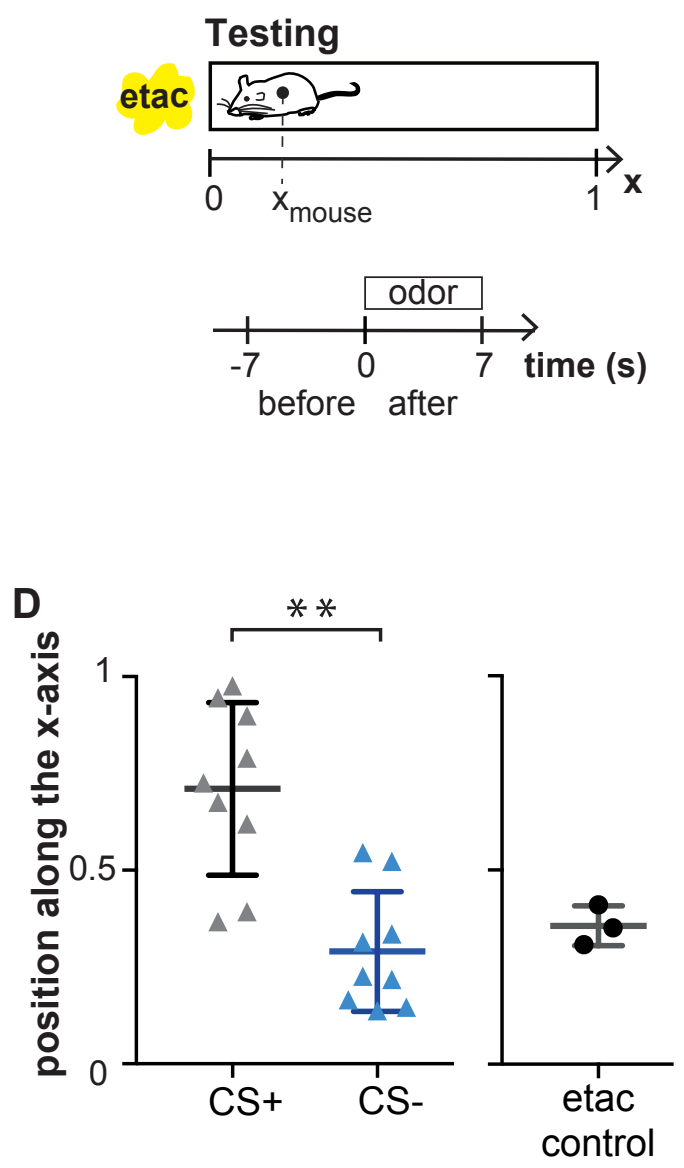

C

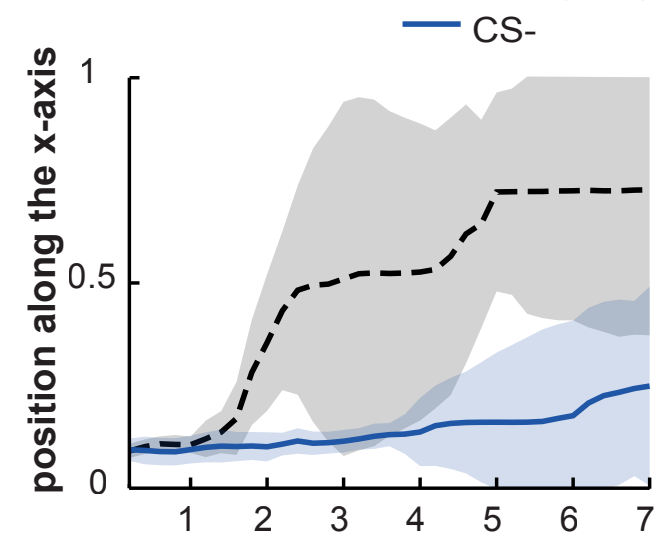

time after odor presentation (s)

E
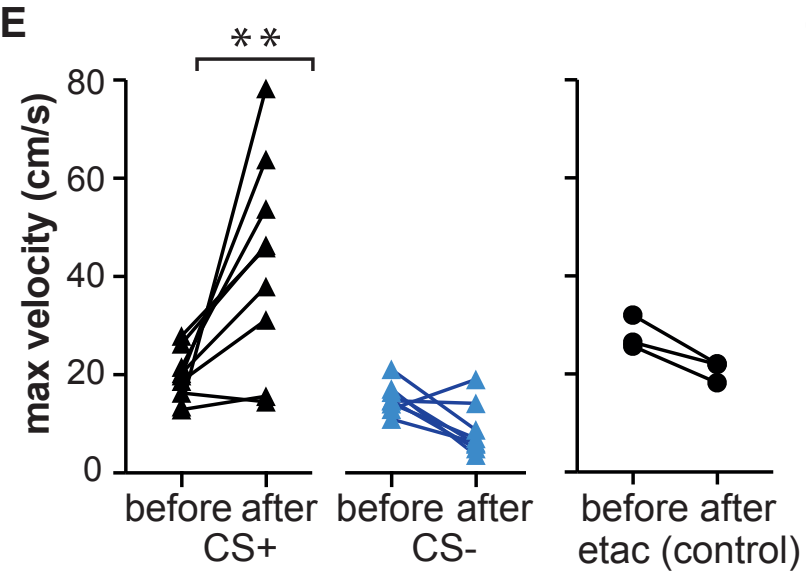

G

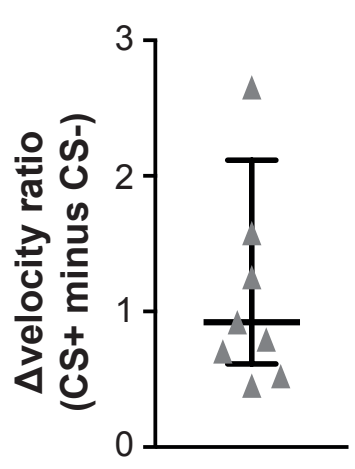

H

$\mathbf{J}$
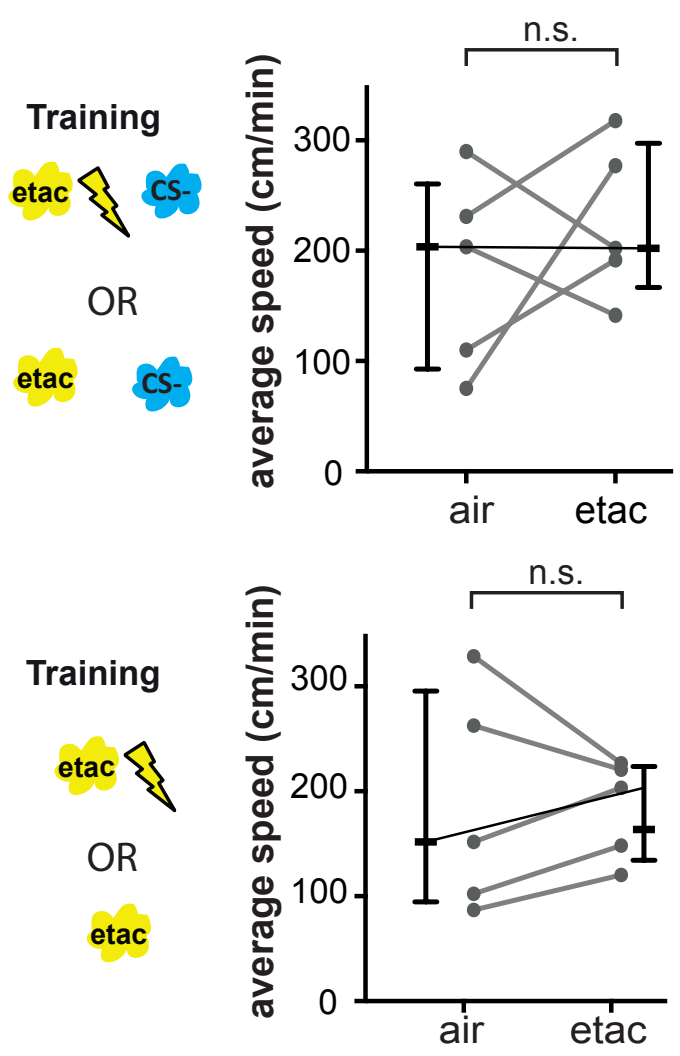
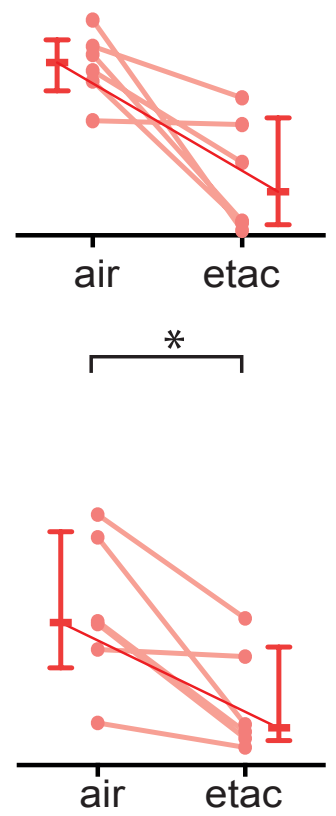

I

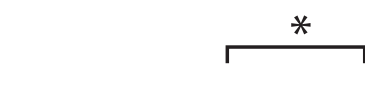

- etac, CS-

- etac+shock, CS-
K

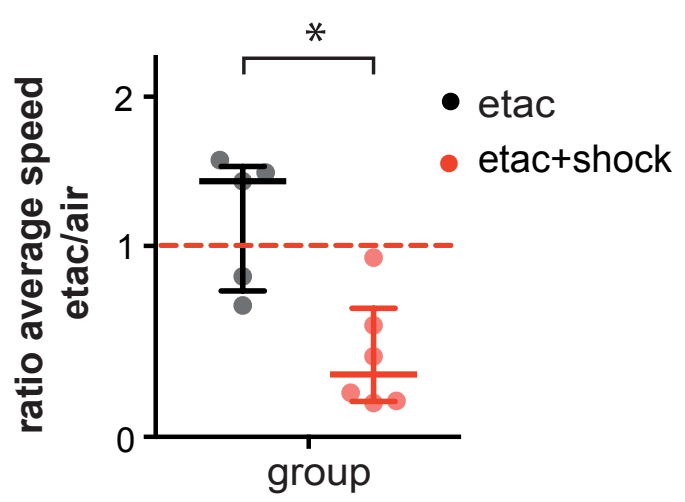


A
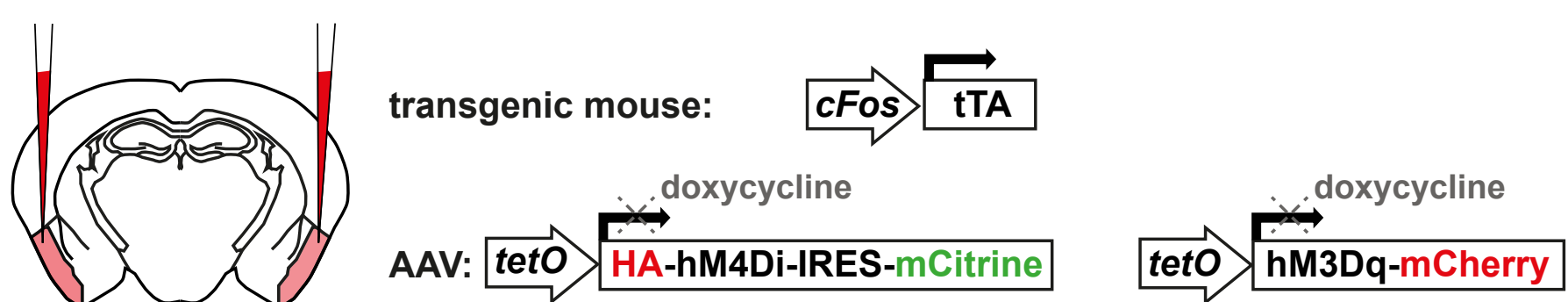

piriform

B

Odor fear conditioned

(CS+, 2 CS-)

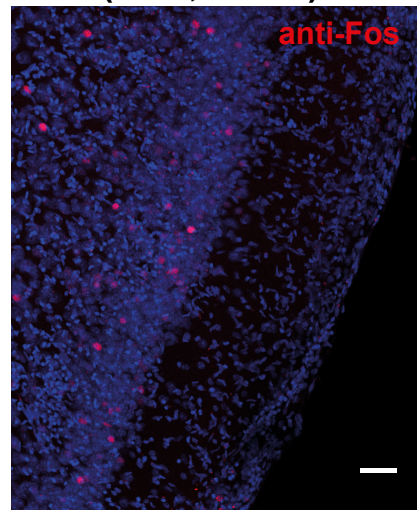

$2.9(0.2) \%$

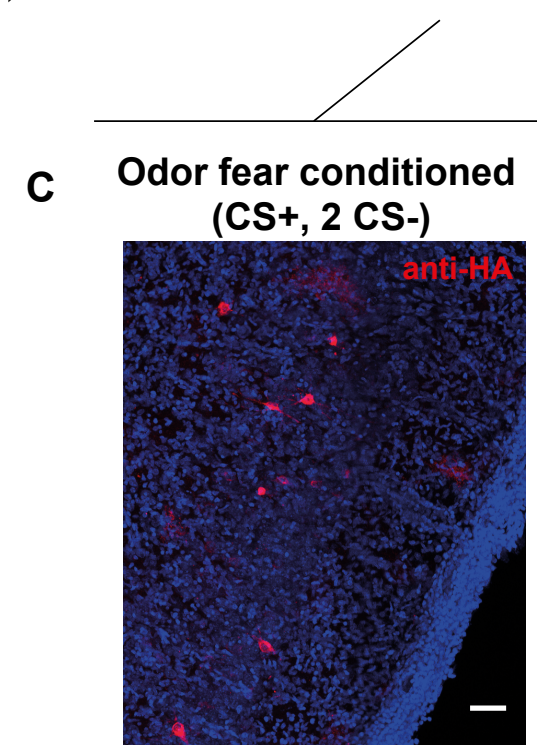

$1.6(0.3) \%$

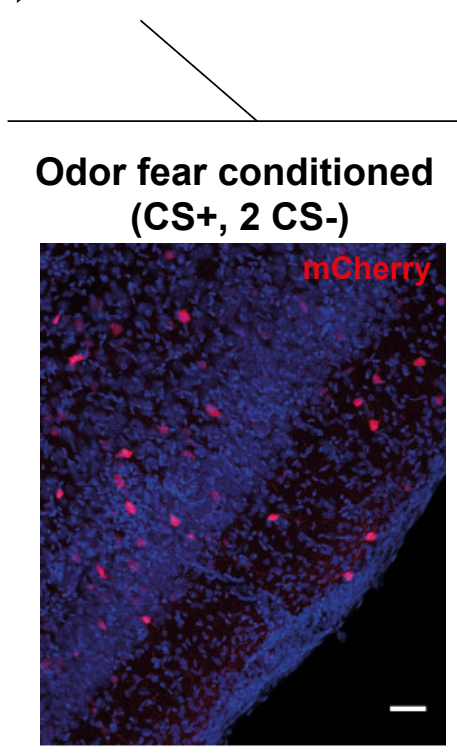

$2.2(0.5) \%$
E

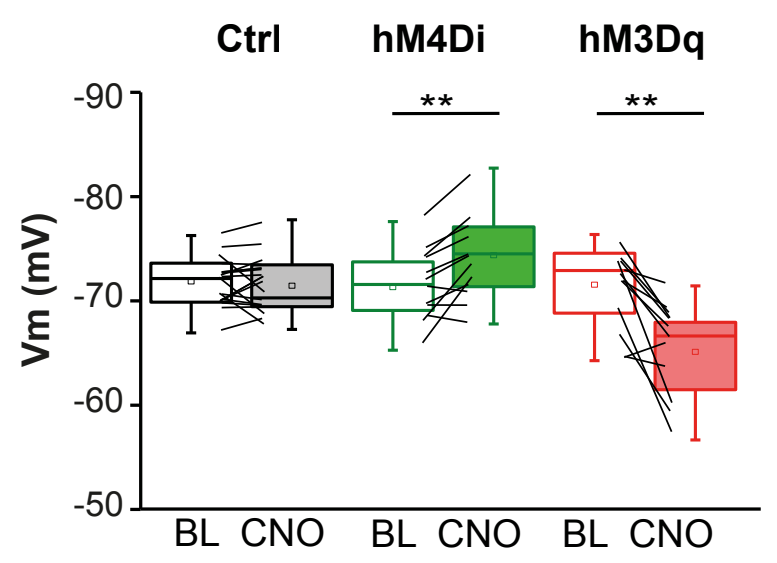

$\mathbf{F}$

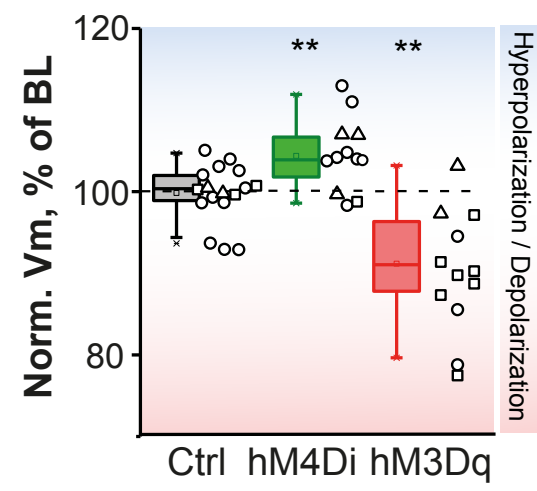

○ $P N$

$\triangle \mathrm{SL}$

a FS

G

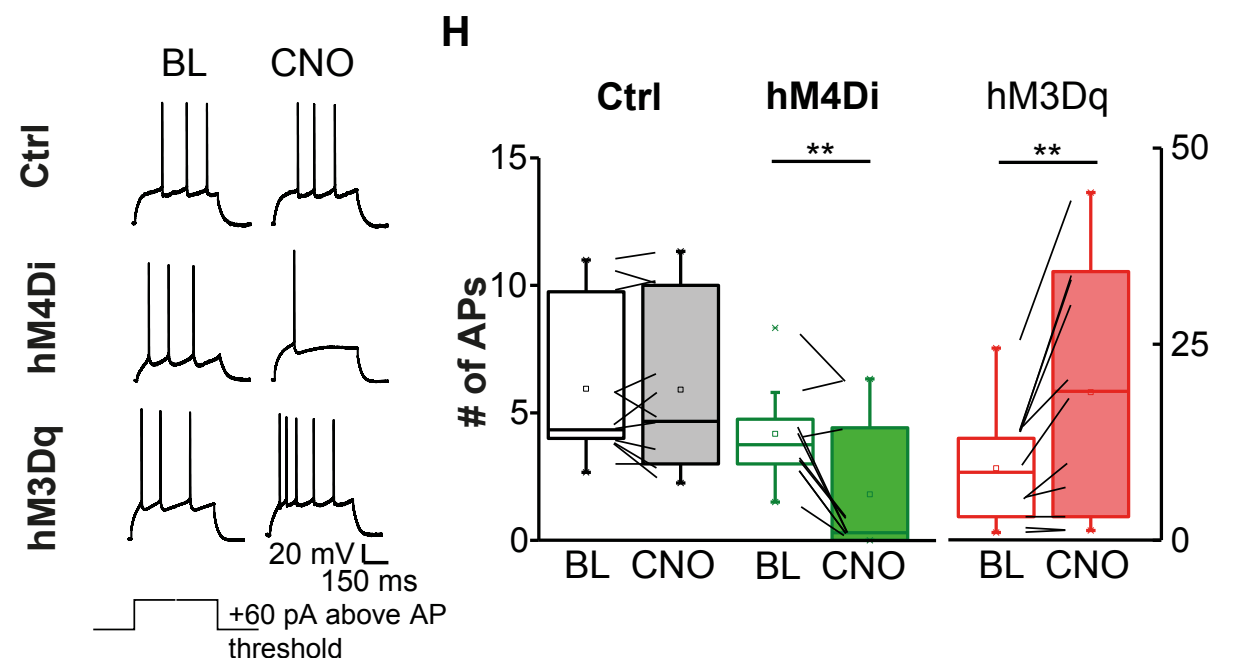

I

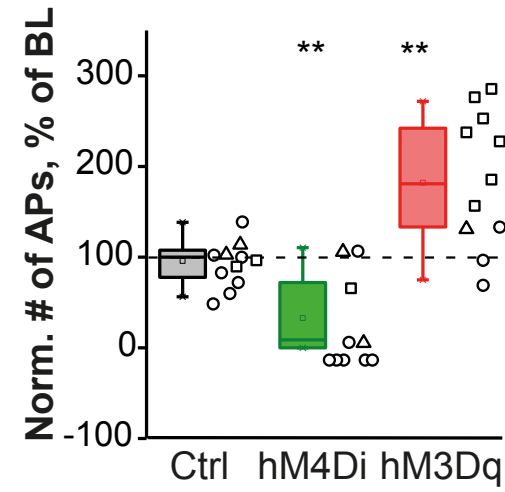




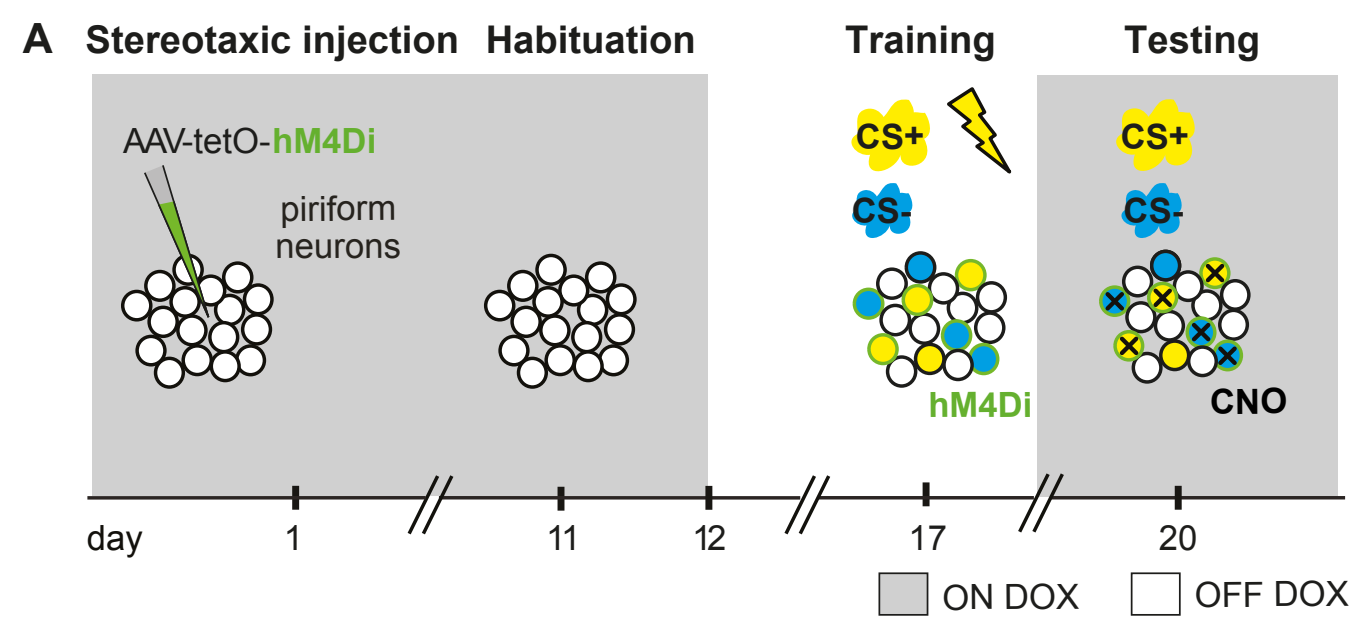

- control group $(n=8)$

- $\exp$ group $(n=8)$
B

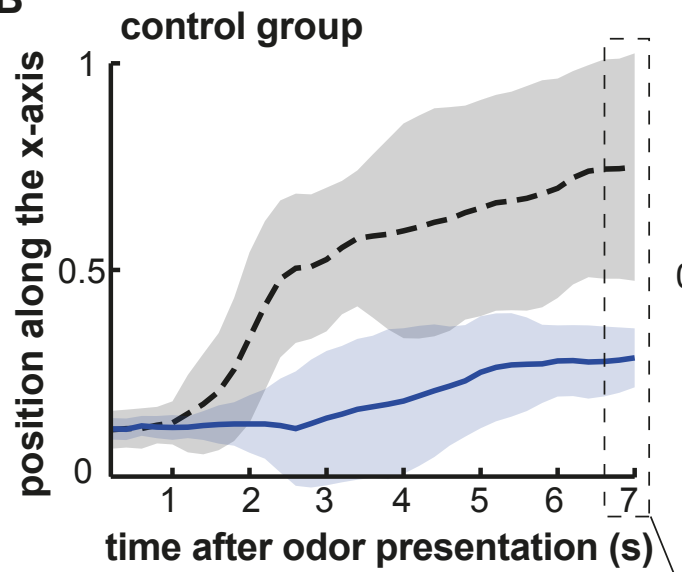

C

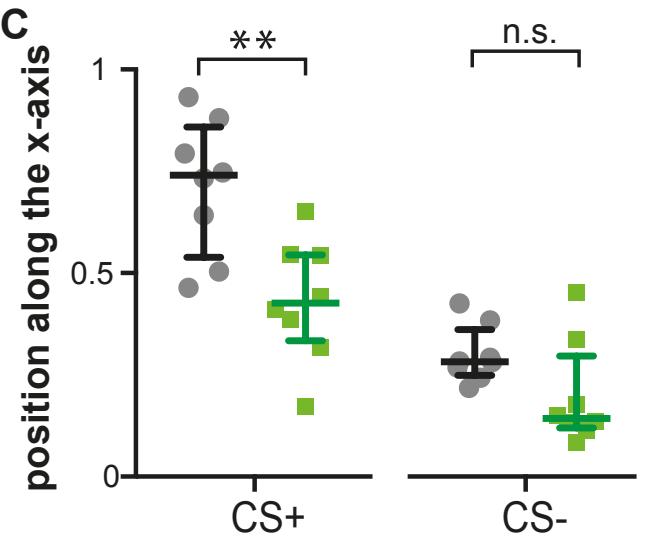

experimental group

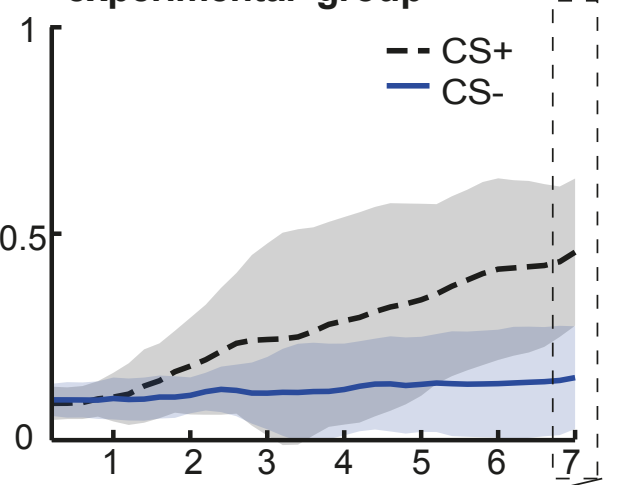

time after odor presentation (s)

D

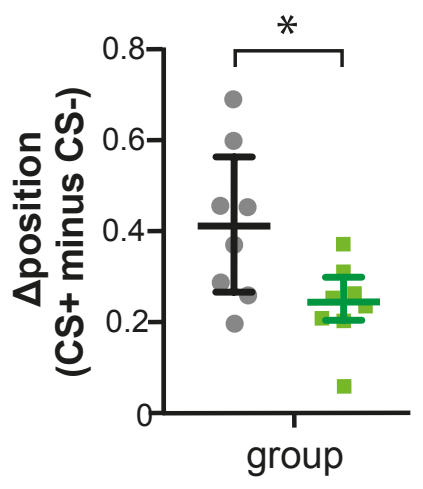

E
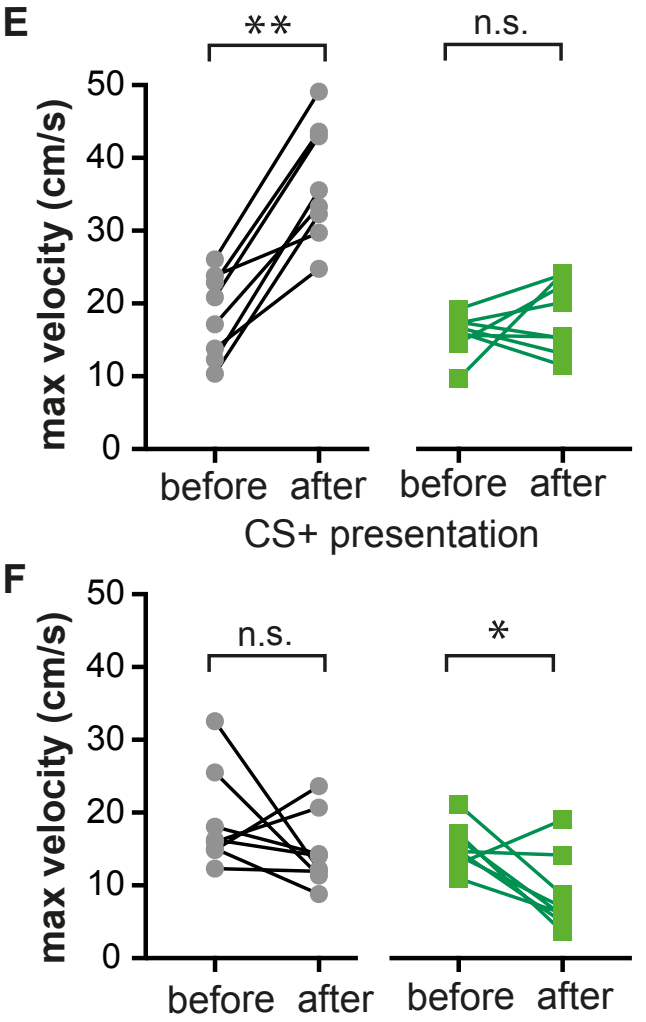
CS- presentation

G

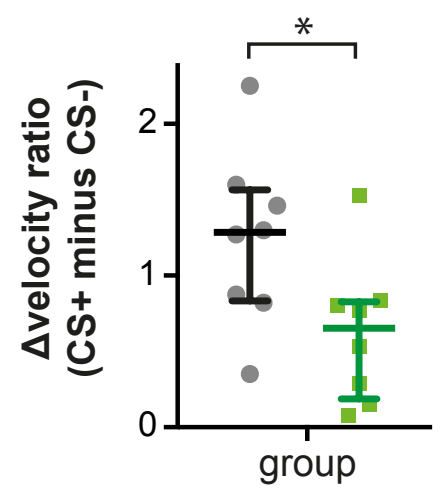


A Stereotaxic injection Habituation
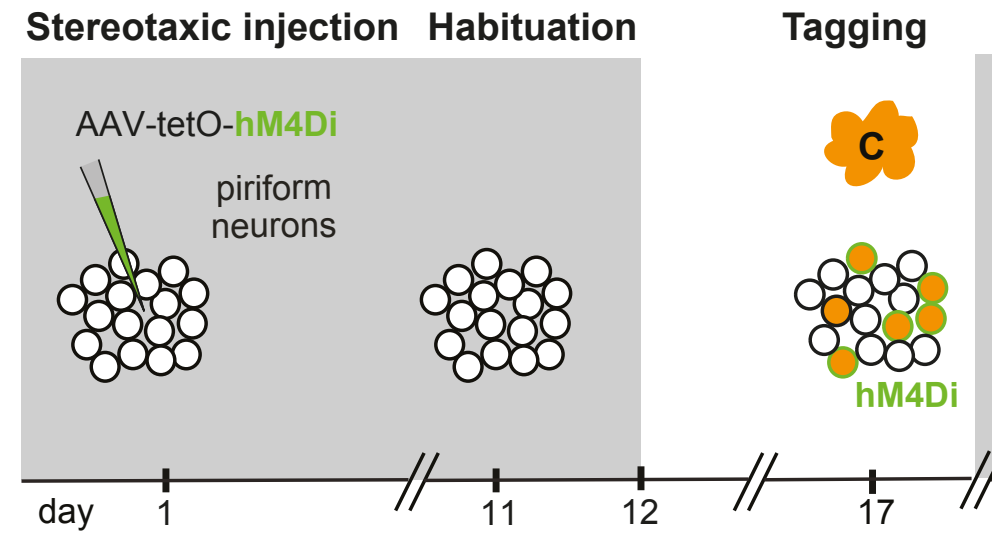

$B$

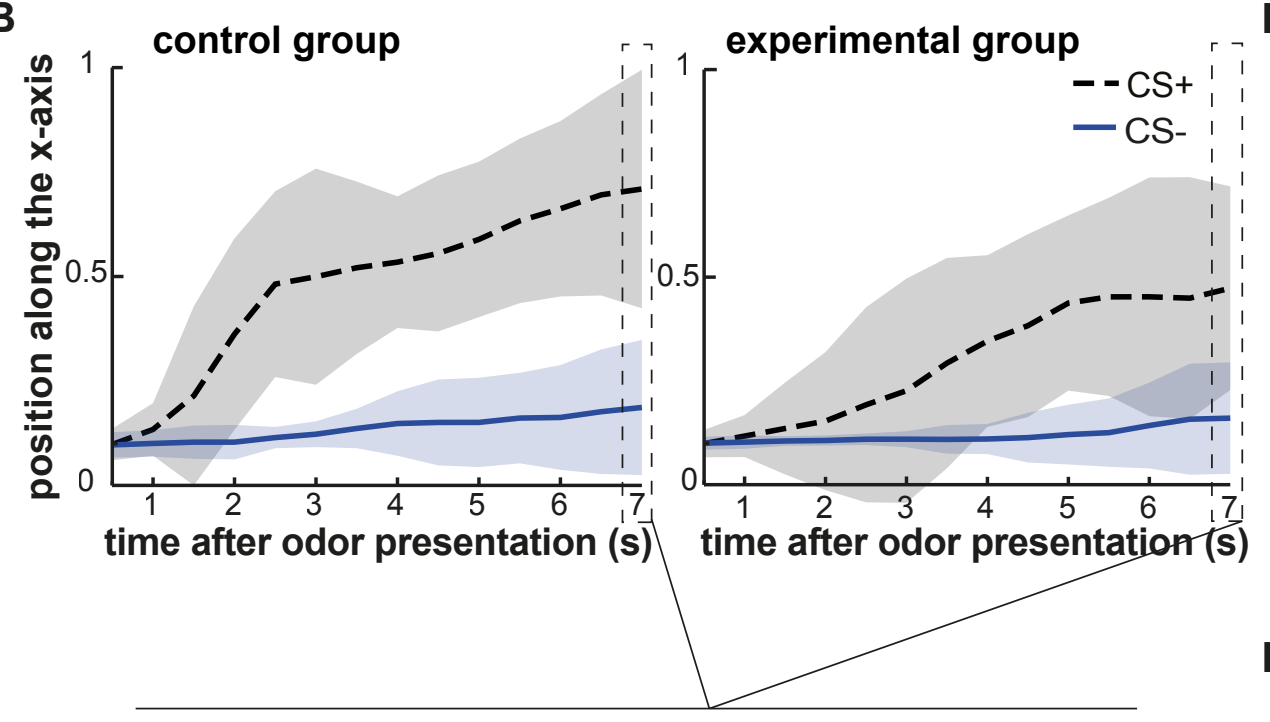

C

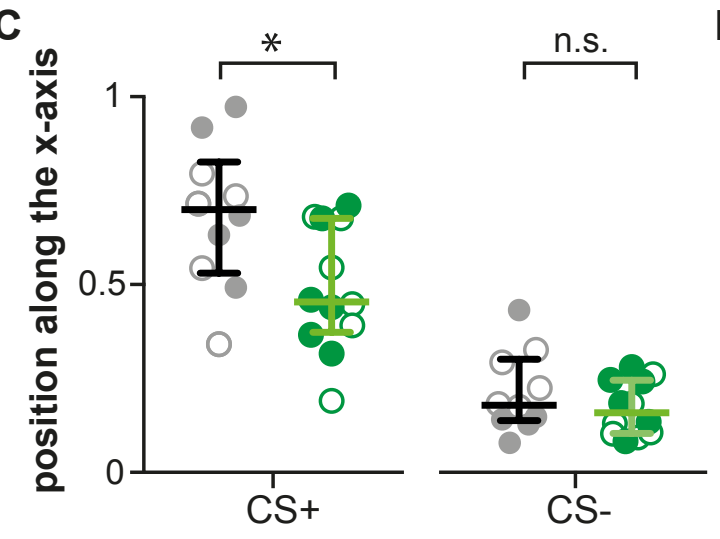

D

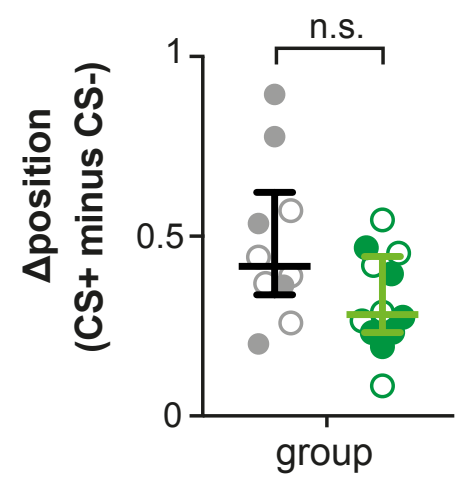

Testing

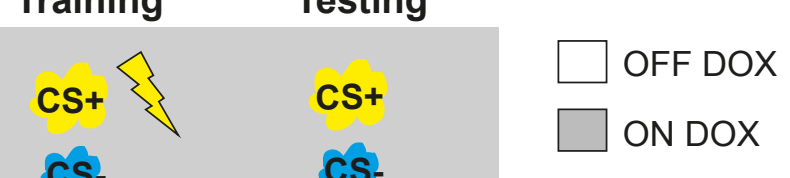

ON DOX

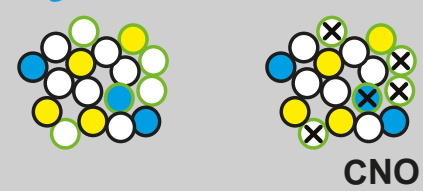

CNO

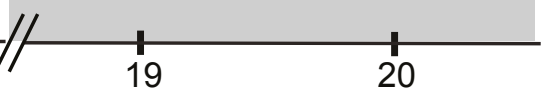

E

control group

o eug. $(n=5)$

exp group

- beta-cit $(n=5)$

o eug $(n=6)$

- beta-cit $(n=6)$

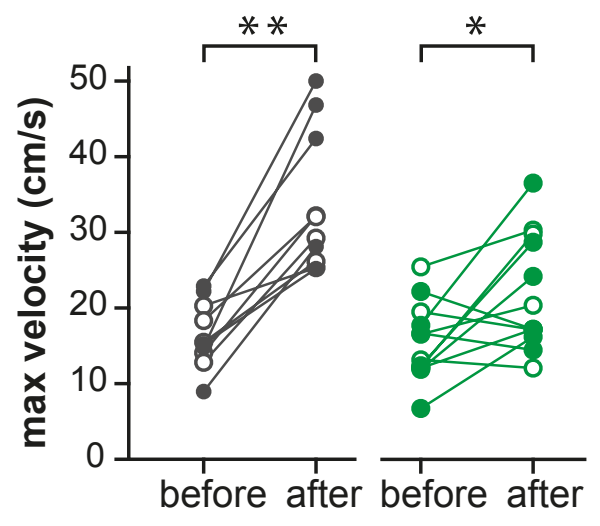

CS+ presentation

F

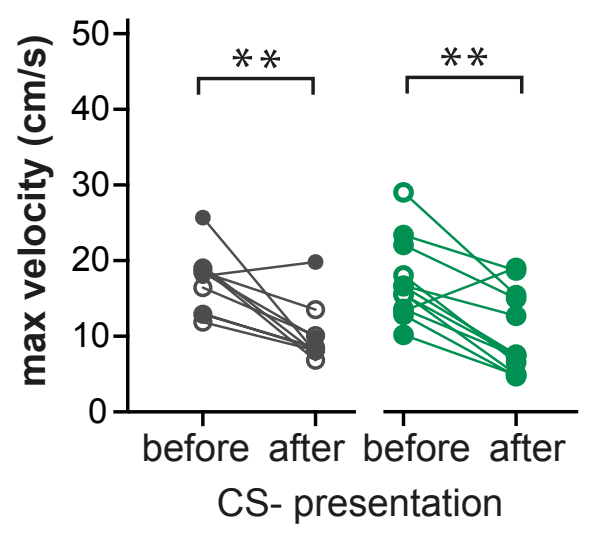

G

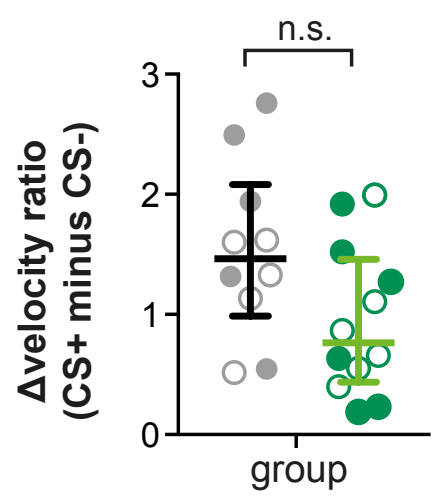


A

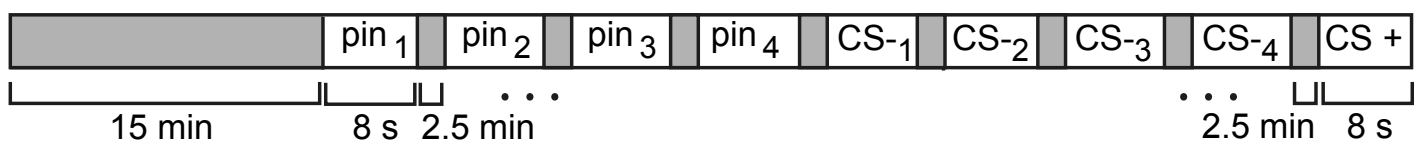
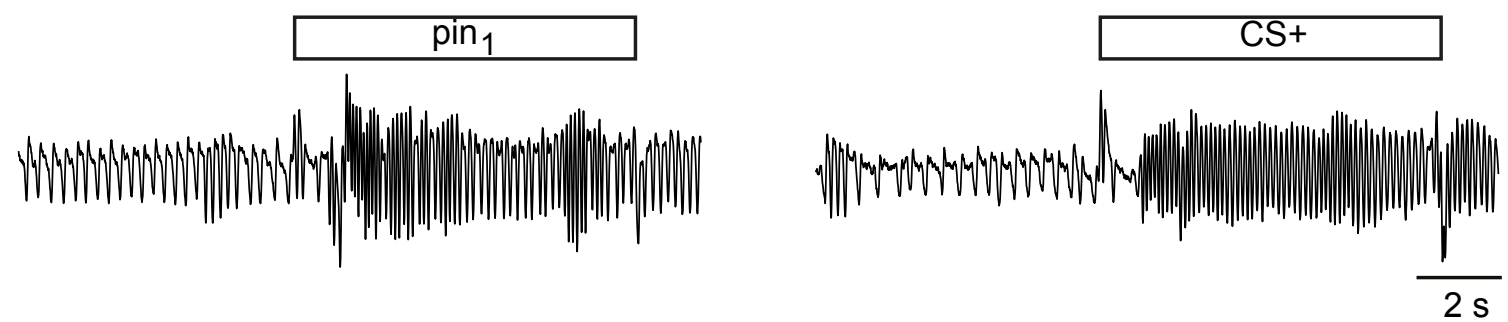

B

C $\stackrel{\text { n.s. }}{\longmapsto}$
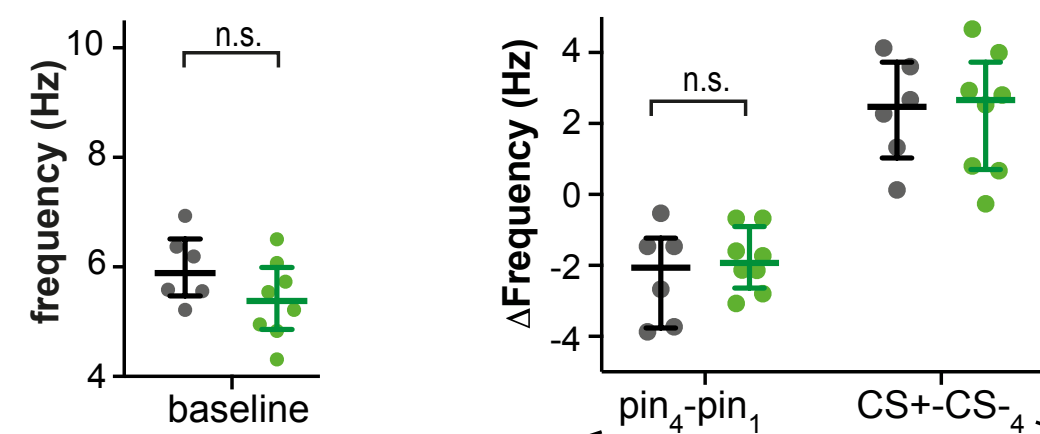

- control group $(n=6)$

- experimental group $(n=8)$
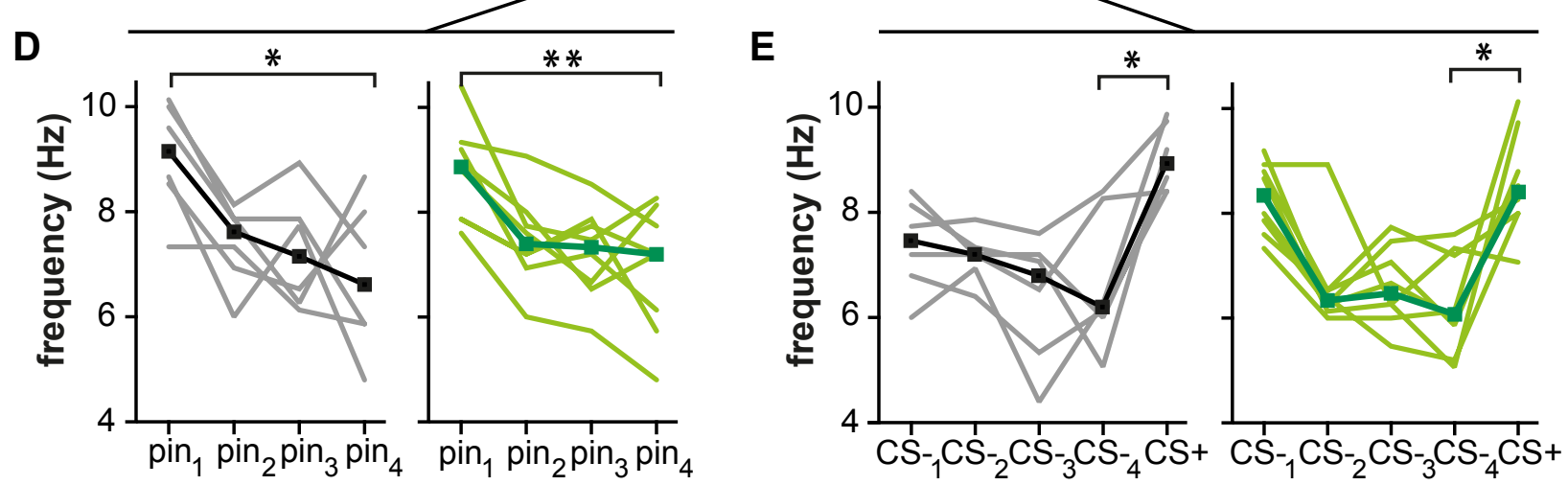
A Stereotaxic injection Habituation

Training

Baseline, open field

Testing, open field

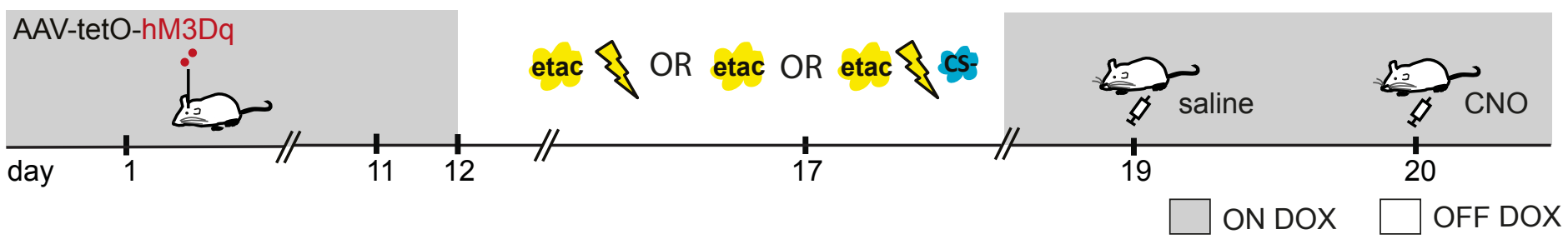

B

- etac

- etac+shock

o-- etac+shock,CS-

C
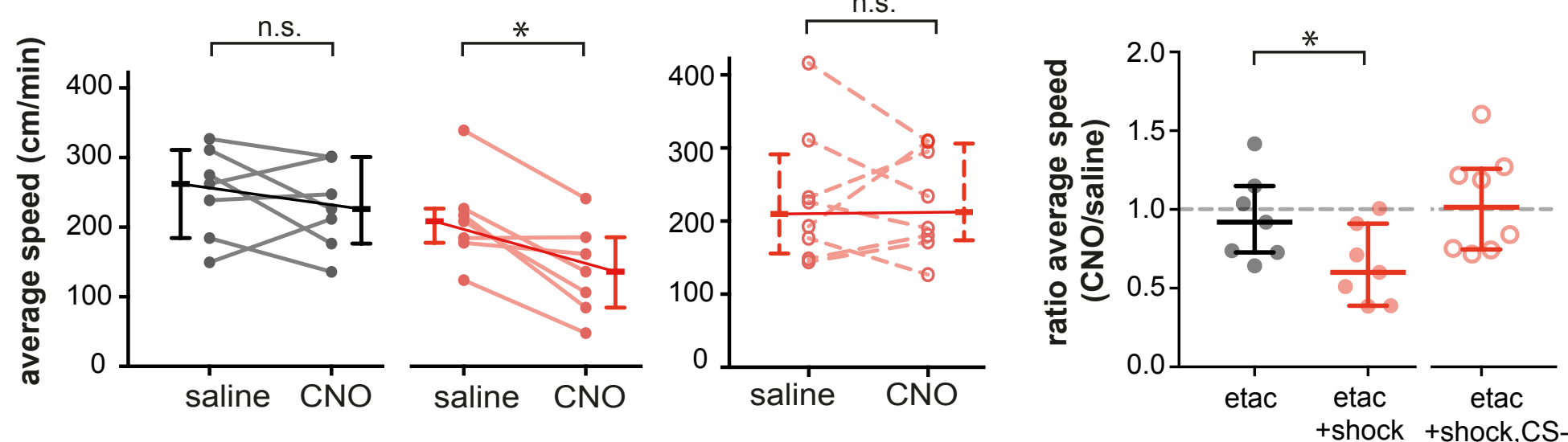